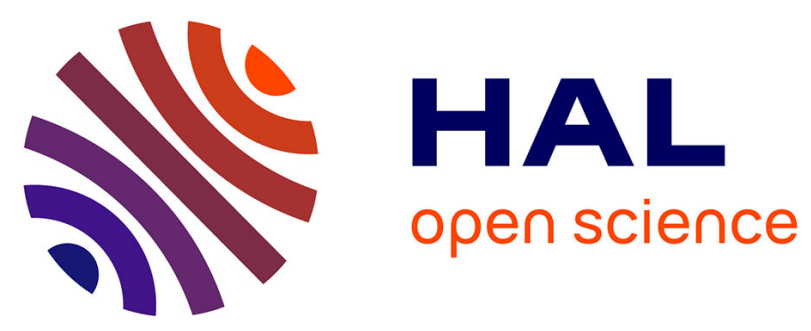

\title{
Numerical Investigations of Perfectly Mixed Condition at the Inlet of Free Radical Polymerization Tubular Microreactors of Different Geometries
}

Dhiraj K. Garg, Christophe Serra, Yannick Hoarau, Dambarudhar Parida, Michel Bouquey, Rene Muller

\section{To cite this version:}

Dhiraj K. Garg, Christophe Serra, Yannick Hoarau, Dambarudhar Parida, Michel Bouquey, et al.. Numerical Investigations of Perfectly Mixed Condition at the Inlet of Free Radical Polymerization Tubular Microreactors of Different Geometries. Macromolecular Theory and Simulations, 2020, pp.2000030. 10.1002/mats.202000030 . hal-02921186

\section{HAL Id: hal-02921186 \\ https://hal.science/hal-02921186}

Submitted on 24 Aug 2020

HAL is a multi-disciplinary open access archive for the deposit and dissemination of scientific research documents, whether they are published or not. The documents may come from teaching and research institutions in France or abroad, or from public or private research centers.
L'archive ouverte pluridisciplinaire HAL, est destinée au dépôt et à la diffusion de documents scientifiques de niveau recherche, publiés ou non, émanant des établissements d'enseignement et de recherche français ou étrangers, des laboratoires publics ou privés. 
DOI: 10.1002/marc.((insert number)) ((or ppap., mabi., macp., mame., mren., mats.))

Full Paper

Numerical investigations of perfectly mixed condition at the inlet of free radical polymerization tubular microreactors of different geometries

Dhiraj K. Garg*, Christophe A. Serra, Yannick Hoarau, Dambarudhar Parida, Michel Bouquey, Rene Muller

Dr. Dhiraj K. Garg

Shiv Nadar University, Dadri, Gautam Buddha Nagar, UP, India-201314

Email: dhiraj.garg@snu.edu.in

Prof. Christophe A. Serra

Université de Strasbourg, CNRS, ICS UPR 22, F-67000 Strasbourg, France

Email: serrac@unistra.fr

Prof. Yannick Hoarau

Université de Strasbourg, CNRS, ICUBE UMR 7357, F-67412 Illkirch, France

Email: hoarau@unistra.fr

Dr. Dambarudhar Parida,

Empa, St. Gallen, Lerchenfeldstrasse 5, 9014 St. Gallen, Switzerland

Email : dambarudhar.parida@empa.ch

Dr. Michel Bouquey, Prof. Rene Muller

Université de Strasbourg, CNRS, ICS UPR 22, F-67000 Strasbourg, France

Email: michel.bouquey@unistra.fr, rene.muller@unistra.fr

The numerical investigation of free radical polymerization (FRP) of methyl methacrylate under mixed feed condition at inlet was made for three microtubular reactor geometries, namely straight tube, coiled tube and coil flow inverter reactor (CFIR). The effect of variation of fluid thermo-physical properties (FTPP) (density, viscosity and thermal conductivity) with reaction along with discrete variation of chemical species diffusion coefficient was studied. Predicted values of monomer conversion, number average chain length and polydispersity index were found to be systematically lower for variable FTPP case compared to constant FTPP case. Results clearly showed the importance of modeling the variation of FTPP especially with respect to conversion. One case of varying diffusion coefficient of each chemical species based on free volume theory with constant FTPP was also simulated. Contrary to expectations, results were found to be independent of reactor geometry for all above cases.. Constant thermal 
conductivity and isothermal reactor condition were found to be good assumptions. Chaotic advection was observed in CFIR under steady-state condition even at very low inlet $\mathrm{Re}=0.06$. This study clearly established the need for modelling coupled transport processes with varying diffusion coefficient along with conversion in modeling of FRP in microreactors for better predictions.

FIGURE FOR ToC_ABSTRACT

CFD Analysis- CFIR, $\left(D=5 \times 10^{-11}\left(\mathrm{~m}^{2} / \mathrm{s}\right)\right.$, variable $)$ Streamlines and velocity pattern for massless tracer particles

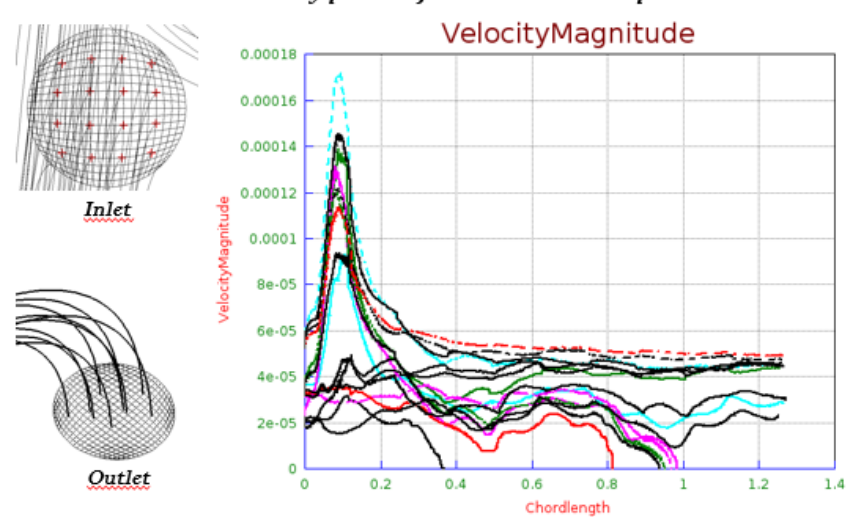




\section{Introduction}

Since mixing has a profound impact on polymerization and polymer product properties, ${ }^{[1]}$ the study of the feed mixing conditions - completely mixed or completely unmixed feed at reactor inlet, is important. In flow reactors, active or passive mixing methods ${ }^{[2]}$ are used for ensuring continuous mixing along the flow. Active methods are energy intensive and require physical devices. Passive methods, on the other hand, induce the mixing by creating variation in flow profile through reactor geometry. They are thus less energy intensive and have little maintenance due to absence of moving components. In microreactor, diffusion process contributes significantly to mixing besides convection mixing ${ }^{[3,4]}$ which is otherwise insignificant in macro-scale industrial reactors. ${ }^{[5]}$ Polymerization in microreactor is a difficult process to carry out due to very large increase of viscosity with conversion. To keep it manageable, non-reactive solvent is generally added to dilute the reacting solution.

Computational fluid dynamics (CFD) can be used judiciously to study, evaluate and compare various microreactor geometries and to choose the best among them and then optimize it but this requires good mathematical modeling of polymerization. A mathematical model is being judged for its goodness by its predictions compared to experimental data under similar operating conditions. The question that how much comprehensive a mathematical model should be, without becoming unnecessarily complex and computationally expensive to give acceptable predictions, is really difficult. Some researchers ${ }^{[4]}$ modelled fluid thermo-physical properties (FTPP) as constant while other ${ }^{[5]}$ had modelled the variation of FTPP during polymerization simulation while all of them kept diffusion coefficient of various chemical species constant. Keeping diffusion coefficient constant in modelling of polymerization is good when radial Peclet number is high $(>100)$ as generally is the case with industrial scale reactors of larger dia. ${ }^{[5]}$ The same is not true for the case of microreactor, especially during lower conversions, where the radial Peclet number could be low $(<100)$. Low Peclet number implies that radial 
diffusion is significant and must be accounted for while predicting mixing. Thus, modelling continuous variation of diffusion coefficient seems to be necessary to evaluate mixing during polymerization inside microreactors.

This leads to another two possibilities of modelling polymerization in microreactors modelling constant or variable FTPP alongwith continuous variation of diffusion coefficient with conversion. Thus, there are four possibilities in all for modelling polymerization in any microreactor- constant or varying diffusion coefficient in combination with constant or variable FTPP. Each possibility requires different amount of computational resources based on complexity of modelling. The modelling with constant diffusion coefficient would require multiple simulations with different values of diffusion coefficient whereas with varying diffusion coefficient, only one simulation is enough. This study actually looks into this issue for existing and proposed improved modeling of free radical polymerization in tubular microreactors under mixed feed conditions at reactor inlet.

The effect of unmixed feed condition on free radical polymerization (FRP) in microreactors of various geometries had already been investigated numerically by some researchers. ${ }^{[3,4]}$ They had also compared those geometries for the mixing and polymer properties under unmixed feed condition. They had done all their CFD modeling and simulation with the assumption of constant FTPP during reaction. This assumption led to decouple the flow process from chemical reaction, mixing and heat transfer. This shortcoming was removed to some extent in our previous work. ${ }^{[6]}$ Two reactor geometries namely straight tube (STR) (Figure 1(b)) and coiled flow inverter reactors (CFIR) (Figure 1(f)) were considered. The feed condition was completely unmixed at reactor inlet. The processes were modeled along with variation of density, viscosity and thermal conductivity as function of conversion and temperature (variable FTPP). This coupled the flow process with heat transfer, mass transfer and chemical reactions - conditions closer to reality. A new transformation (NT), developed in another work, ${ }^{[7]}$ was 
used. The process conditions were still unmixed feed condition of FRP in straight tube (STR) and coiled flow inverter (CFIR) tubular microreactors. This allowed feeding the CFD code for FRP with data in molar form for chemicals species concentration and kinetic rates coefficients instead of in mass form as used by other researchers. ${ }^{[3,4]}$ It was found that this approach reduced numerical and modeling errors significantly and hence improved the predictions of FRP using CFD in microreactors.

The simulations were carried out against the discrete variation of diffusion coefficient with its value being same and constant for all the chemical species modeled. Its effect was observed on several variables like monomer conversion $X_{M}$, polydispersity index $(P D I)$ and number-average polymer chain length $(D P n)$. The discrete variation was considered because of the difficulty to model continuous variation of diffusion coefficient for each chemical species. Although this approach was quite simplistic in nature, still it gave some understanding of the process inside microreactor. Its effect was observed on several variables like monomer conversion $X_{M}$, polydispersity index (PDI) and number-average polymer chain length $(D P n)$ at reactor outlet. Results were then compared with the constant FTPP modeling case under similar conditions. Two reactor geometries namely straight tube (STR) and coiled flow inverter (CFIR) reactors were considered. CFIR is a chaotic flow device (Figure 1 (f)) which was found to significantly increase internal mixing owing to $90^{\circ}$ bends placed at regular intervals along the helix. ${ }^{[8]}$ Significant differences were found in the prediction of $X_{M}, D P n$ and PDI after coupling the processes compared to decoupled processes case of constant FTPP. Viscosity was found to rise by about 6 orders from inlet to outlet and had profound impact on flow profiles in coupled processes case. As a consequence, mixing due to convective flow was severely affected. An anomalous case of increased convective mixing at low diffusion coefficient was observed which could not otherwise be simulated and observed using constant FTPP. Thermal conductivity was modeled as a function of $X_{M}$ but no significant variation in its value was observed. The 
temperature profile across cross-section of the microreactor was found to be uniform indicating existence of isothermal condition even under the varying FTPP condition. CFIR was found to perform better along with an improved control over polymer properties (lower PDI, higher DPn) compared to STR for unmixed feed under similar operating conditions. CFIR, a chaotic flow device, was found to significantly increase internal mixing at industrial scale owing to $90^{\circ}$ bends placed at regular intervals along the helix. ${ }^{[8]}$ Here too, CFIR was found to perform better along with an improved control over polymer properties (lower PDI, higher $D P n$ ) compared to STR under similar operating conditions for unmixed feed.

CFIR seemed to be a promising microreactor for FRP but required more thorough and rational analysis of its performance against simpler and similar geometries. Besides this, completely mixed feed condition at reactor inlet was general practice in labs and industry. So the current study considered the case of mixed feed condition for the FRP of methyl methacrylate (MMA). Three different tubular microreactor geometries were taken for current study. The flow was taken to be laminar inside the microreactors. There was a gradual change in the geometry of each microreactor for enhancing passive radial mixing. ${ }^{[2]}$ The first reactor geometry considered was the straight tube (STR, Figure 1 (b)) which relied on radial mixing by mass diffusion only. Second was the coiled tube reactor (CTR, Figure 1 (d)) which took benefit from secondary flows arising from its curvature to increase radial mixing ${ }^{[9-12]}$ in addition to diffusion. The third reactor geometry was the CFIR (Figure 1 (f)) which presented $90^{\circ}$ bends in an helically based geometry.

In current work, the improvement in mixing, control of polymer properties like PDI and DPn due to reactor geometry and coupling of various transport processes along with chemical reaction were studied and evaluated in a more systematic way. For this, two different types of modelling were used. For the first type of constant diffusion coefficient, like in our previous work, ${ }^{[6]}$ two sets of simulations - one for coupled transport processes (through variable FTPP) 
and another for decoupled transport processes cases (through constant FTPP) - were obtained for all the three microreactor geometries and results were then compared. This resulted in several simulations for different values of diffusion coefficient. For the second type of varying diffusion coefficient, for the first time, for all the three reactor geometries under same operating conditions, one simulation with modeling of continuous variation of diffusion coefficient for all chemical species of FRP using free volume theory ${ }^{[13-15]}$ was made while keeping FTPP constant. This was done to verify whether this would make the modeling sufficiently comprehensive, without becoming too complex and difficult to simulate, to give acceptable predictions.

\subsection{Mathematical Model for CFD}

Following equations were used for CFD problem with chemical reaction and heat effects:

The conservation of Mass (incompressible fluid)

$\nabla \cdot u=0$

The conservation of Momentum - Navier-Stokes equation

$\rho \frac{\partial u}{\partial t}+\rho(u \cdot \nabla) u=-\nabla p+\nabla \cdot\left(\eta\left[\nabla u+(\nabla u)^{T}\right]\right)$

The conservation of Energy with heat generation Q

$\rho C_{p} \frac{\partial T}{\partial t}+\nabla \cdot(-K \nabla T)=Q-\rho C_{p} u \cdot \nabla T$

where $Q=-\Delta H_{p} K_{p} \lambda_{0} M$

The conservation of Chemical species

$\frac{\partial C_{i}}{\partial t}+\nabla \cdot\left(-D_{i} \nabla C_{i}+C_{i} u\right)=R_{i}$

The chemical species were modeled as passive scalars and the generation term $R_{i}$ (Equation 5) for each chemical species is presented in next section.

\subsection{Kinetic Model of FRP}

The various steps of kinetic model considered ${ }^{[6]}$ in this work are given in Scheme 1. 
Initiator decomposition

Initiation

Propagation

Termination by combination

Termination by disproportionation

Transfer to monomer

Scheme 1- Kinetic scheme for free radical polymerization as used in this work

\subsection{Mathematical Model of FRP}

The mathematical model ${ }^{[16]}$ studied in this work was based on the method of moments. $\lambda_{0}, \lambda_{1}$ and $\lambda_{2}$ were zeroth, first and second order moments of the live polymer chain length distributions whereas $\mu_{0}, \mu_{1}$ and $\mu_{2}$ were zeroth, first and second order of moments of dead polymer chain length distribution. The model is presented in Appendix-A in Supplementary Information.

\subsection{New Transformation}

The new transformation (NT) used in this work is given in Appendix-B in Supplementary Information. For details, please refer to our previous work. ${ }^{[7]}$

\subsection{Physical and Chemical Data}

Physical and chemical data used for MMA were taken from various references. ${ }^{[5,17-24]}$

\section{Methodology}

\subsection{Numerical Modeling}

The residence time within all the flow microreactors were kept at about $12 \mathrm{hrs}$. A reputed and proven commercial CFD software CFD-ACE+ was used. ${ }^{[6,7]}$ CFD-GEOM was used for geometry and grid generation. It worked for both structured and unstructured grid as shown in our previous work. ${ }^{[6,7]}$ Structured grid was used in this work for all geometries. Detailed mesh 
independency test was carried out presented in a later section. CFD-ACE was used for CFD simulations and the flow, heat and scalar modules available in it were used for the same. CFDVIEW was used for post-processing of all the simulations.

Five chemical species whose generation terms were represented by Equation (A1), (A2), (A9) - (A11) were modeled as passive scalars, denoted as Scalar1-5 respectively. The spatial distribution of velocity and all the five scalars were taken to be 3rd order so as to improve the numerical accuracy and to reduce the numerical diffusion in the simulations. Conjugated gradient squared (CGS) + Preconditioner solver was selected for velocity, enthalpy and all five passive scalars whereas Algebraic Multigrid (AMG) solver was selected for pressure. SIMPLEC algorithm was used for pressure-velocity coupling in the CFD code. Relaxation parameters for various variables like velocity, pressure, passive scalars etc. were adjusted to ensure convergence of the simulation. The simulations were assumed to be converged when the residual error ratio reduced below $10^{-8}$ for all the variables. The relaxation parameters were also adjusted to increase the rate of convergence and to reduce the simulation time. Flow-average values of all the variables were evaluated at reactor outlet.

The reaction conditions and reactor operating conditions as given in Table $\mathbf{1}$ were same as they were taken in our previous work ${ }^{[6]}$ except for the feed condition at reactor inlet. In current study, the feed was fully mixed in terms of initiator and monomer concentration with $30 \%$ dilution by solvent at inlet. The feed condition for all the cases was considered same. At the inlet, velocity profile was taken to be parabolic to model fully developed flow. The concentration profiles of various chemical species were taken to be flat to model fully mixed feed condition. The inlet temperature of the feed to the reactor was taken to be same as isothermal wall temperature of the reactor i.e. $70^{\circ} \mathrm{C}$. No-slip at reactor wall, zero flux for all the passive scalars across the wall and isothermal condition at reactor wall were taken as boundary conditions. Due to NT, the 
values of scalars were normalized. Hence the values of Scalar 1 and 2 were taken to be 1 and Scalar 3-5 were taken to be 0 at inlet, i.e. only initiator and monomer were present in the feed. Two sets of simulations were conducted : one for coupled transport processes by modeling variation of FTPP and another was decoupled transport processes modeled through constant FTPP. This was done to evaluate the effect of coupling the transport processes over the results obtained through decoupled transport processes case. These simulations were carried out against the discrete variation of diffusion coefficient as it was done in our previous work. ${ }^{[6,7]}$ The models for variation of density, viscosity and thermal conductivity were taken from Baillagou et al. ${ }^{[5]}$

One simulation with continuous variation of diffusion coefficient with conversion for different chemical species with constant FTPP was also carried out for each microreactor geometry for same operating conditions.

The continuous variation in diffusion coefficients was modeled using free volume theory. The theory for calculating diffusion coefficient was developed by Vrentas and Duda ${ }^{[14,15]}$ for predicting diffusion coefficient. This was the most successful theory for predicting diffusion coefficient so far. Expressions to calculate these diffusion coefficients are given below, details of which could be found elsewhere. ${ }^{[13]}$

$$
\begin{aligned}
& D_{M}=D_{M 0} \exp \left[-\frac{\gamma_{M} \xi_{M P}}{V_{f}}\left(\frac{\omega_{M} V_{M}^{*}}{\xi_{M P}}+\omega_{P} V_{P}^{*}+\frac{\omega_{S} V_{S}^{*}}{\xi_{S P}}\right)\right] \\
& D_{P}=\frac{D_{P 0}}{\left(\frac{M W_{M}^{2}}{M W_{M}}\right)} \cdot \exp \left[-\frac{\gamma_{P}}{V_{f}}\left(\frac{\omega_{M} V_{M}^{*}}{\xi_{M P}}+\omega_{P} V_{P}^{*}+\frac{\omega_{S} V_{S}^{*}}{\xi_{S P}}\right)+\frac{\gamma_{P}}{\xi_{M P} V_{f M}}\right]
\end{aligned}
$$

where:

$$
\begin{aligned}
& D_{P 0}=\frac{k_{B} T}{6 \pi \eta_{M}\left(R_{H}\right)_{0}} \\
& \left(R_{H}\right)_{0}=\left(\frac{3}{10 \pi N_{A}}[\eta]_{0} M W_{M}\right)^{\frac{1}{3}} \\
& {[\eta]_{0}=K_{M H} \cdot M W_{M}^{a_{M H}}}
\end{aligned}
$$


$V_{f}=\omega_{M} V_{M}^{*} V_{f M}+\omega_{P} V_{P}^{*} V_{f P}+\omega_{S} V_{S}^{*} V_{f S}$

$\xi_{k P}=\frac{M_{j k} V_{k}^{*}}{M_{j P} V_{P}^{*}}$

The effect on various reaction parameters like $X_{M}, P D I$ and $D P n$ with discrete variation in diffusion coefficient of chemical species was studied. During discrete variation of diffusion coefficient among simulations, the value of diffusion coefficient was assumed to be constant and same for all the passive scalars (chemical species) in a given simulation. For continuously varying diffusion coefficients case, first two passive scalars $-1 \& 2$, representing initiator (in solvent) and monomer, were considered to have same value of diffusion coefficients and thus calculated using Equation (6). The value of diffusion coefficients for the rest three passive scalars was considered same as these scalars represented dead polymer chains and were calculated using Equation (7).

\subsection{Meshing}

CFD-GEOM was used to generate the reactor geometry. A specific program was developed to generate CFIR of desired geometrical specification including pitch, number of turns in between the two bends, number of bends, curvature ratio and number of grid points in axial flow. Dimensions of all the three reactors were kept identical especially in terms of the length to have identical residence time and number of turns in integral value in both CTR and CFIR to the extent possible (Table 2). Structured grid was used for all the geometries. Square mesh was selected for circular cross-section. Only the upper half was modeled for STR due to axial symmetry but its cross-sectional mesh density was kept same as that of CTR and CFIR. The structured mesh and geometry of the three reactors were shown in Figure 1.

\subsection{Simulation}

In our previous work, ${ }^{[16]}$ an analytical solution (AS) of the set of differential equations constituting the mathematical model of FRP (as given in Appendix-A) was derived for ideal batch reactor conditions, i.e. isothermal, well mixed, batch reactor solution 
homopolymerization before gel/glass/cage effect. To check the correctness of coding of mathematical model along with NT, STR with coarse meshing was first simulated as batch reactor with same operating conditions already mentioned except for no flow at inlet and outlet. The values of Scalar 1 and 2 were taken to be 1 and the value for Scalar 3-5 was taken as 0 each for the whole volume as the initial value for the simulation. The simulation was transient in nature with the total time of simulation kept same as residence time. Its results were compared with AS under same conditions.

The batch reactor results with time were theoretically applicable for the plug flow reactor with distance along flow. So, STR was then simulated as plug flow reactor (PFR) with coarse meshing by imposing flat velocity and flat concentration profile at inlet and same inlet velocity at wall instead of no-slip as boundary condition. The other operating conditions were kept same as already mentioned. The results were again compared with AS under similar conditions. For both above tests, the data taken for monomer was for styrene. For details about its chemical data, please refer to our previous work. ${ }^{[6,7]}$ Both these test simulations would validate the coding of the mathematical model in CFD beyond doubt.

After this, extensive mesh independence tests were carried out for all the three reactor geometries individually. Extensive mesh independence analysis was done for a given reactor geometry for fully mixed feed condition. For each reactor geometry, three different diffusion coefficients $\left(D=1 \times 10^{-12}, 1 \times 10^{-10}, 1 \times 10^{-8} \mathrm{~m}^{2} / \mathrm{s}\right)$ were used to cover the complete range of variation of diffusion coefficient under study. Mesh density was changed in both radical and axial directions and then it was checked for convergence. Finally, only four most suitable cases of grids were selected for further evaluation where all the variables satisfactorily became mesh independent. These four cases arose from the combination of changes in axial direction mesh density and cross-sectional mesh density. All the variables were to be made independent of mesh for that particular mesh to be selected. 
Once the final mesh was selected, all the proposed CFD simulations were then carried out. Same final mesh was used for continuously varying diffusion coefficient case.

\section{Results and Discussion}

The result of STR modeled as batch reactor is shown in Figure 2. The result shows the values of five Scalars plotted on the same axis. Due to NT used, their values are normalized and dimensionless in terms of concentration. It matched exactly with AS. This proves that the code related to implementation of rate equations for scalar 1-5 as source terms in their respective equations, is correctly implemented.

The results for STR as PFR compared to AS are given in Table 3. As can be seen, the results matched excellently with AS obtained under the same reactor conditions. This proved that code related to the scalar1-5 equations was properly integrated with flow and energy equations Thus, both batch type reactor as well as plug flow type reactor simulations validated the correctness of the implementation of the code related to reaction equations of scalar 1-5 within the software for simulation along with the correct implementation of the NT.

Extensive mesh independence analysis was done for all variables. One such variable, monomer conversion $X_{M}$, is shown in Table 4 for the STR only. The same procedure was also used for CTR and CFIR also.

After mesh independence test, a common mesh was found to be suitable for the whole range of variation of values of diffusion coefficient taken under study for a given reactor geometry. In this problem, the values of variables were found to be more dependent upon mesh density in flow direction rather than on cross-sectional mesh density as highlighted by Table 4. The same observation was made for all the three reactor geometries. This helped in selecting coarse mesh density for cross-section to reduce the total number of mesh point without affecting the results. The final mesh selected for a given geometry for mix inlet condition is given in Table $\mathbf{5}$. 
The values of relaxation parameters were found to have huge impact on the rate as well as the level of convergence during our study. But once a given simulation was found to converge (based on residual error ratio value criteria already mentioned), the values of variables in the converged solution were found to be independent of relaxation parameter values. The values of relaxation parameters were found to be affected by the values of diffusivity coefficient. The values chosen for relaxation parameters were found to reduce the residual error ratio value to levels below $10^{-8}$ and thus improving the accuracy of the simulations. Furthermore, they also increased the rate of convergence. The stopping criteria of residual error ratio value was achieved in 150-3000 iterations depending on the simulation.

For constant FTPP case, the convergence was obtained over a large range of diffusion coefficient values ranging from $1 \times 10^{-12} \mathrm{~m}^{2} / \mathrm{sto} 1 \times 10^{-8} \mathrm{~m}^{2} / \mathrm{s}$ for all the three reactor geometries under study. For variable FTPP case, the convergence could be obtained for limited values of diffusion coefficient: in five cases for STR and in just three cases each for CTR and CFIR. Simulations for varying diffusion coefficient could only be converged for the case of constant FTPP case.

Figure 3 shows some important results about monomer conversion, $X_{M}$. For all the three reactor geometries, $X_{M}$ was found to be independent of the geometry for both the cases of constant (decoupled transport processes case) and variable FTPP (coupled transport processes case). Although the trend as well as values of $X_{M}$ differed from each other for these two cases. The geometry independence of $X_{M}$ could probably be due to mixed feed condition which lowered the requirement for mixing within the microreactor compared to unmixed condition as shown in our previous work ${ }^{[6,7]}$ and other published results. ${ }^{[3,4]}$ The values of $X_{M}$ predicted for the coupled transport processes case were lower compared to the one predicted for decoupled transport processes case. Besides this, for decoupled case, the variation in $X_{M}$ value was small over the complete range of variation of diffusion coefficient. Contrary to this, for coupled case, 
there was appreciable decrease in $X_{M}$ when the value of diffusion coefficient was decreased. This seemed to be more realistic as decrease in value of diffusion coefficient would decrease radial mixing. In absence of proper radial mixing, $X_{M}$ in the central portion of the flow would remain low because it would experience less residence time due to higher velocity in and around the center of the tube. This clearly establishes the importance of modeling the variation in FTPP over constant ones. This meant that the modeling of transport processes as coupled one is necessary to obtain physically correct results for FRP in tubular microreactors.

Figure 4 shows the results for number average chain length, $D P n$. It also exhibited geometry independence similar to $X_{M}$ in Figure 3 for both the cases of variable and constant FTPP. With respect to trend, $D P n$ increased as the value of diffusion coefficient decreased for both the cases. In FRP, DPn decreases as $X_{M}$ increases. Decreased diffusion coefficient lowered $X_{M}$ as seen in Figure 3. So, lower $X_{M}$ due to lower diffusion coefficient made $D P n$ to rise. Here again, similar to $X_{M}, D P n$ values were also predicted to be lower in case of coupled transport processes case compared to decoupled transport processes case.

Figure 5 shows the results related to polydispersity index, $P D I$. Here again, the values of $P D I$ were found to be independent of reactor geometry for both the cases of constant and variable FTPP. The predictions of coupled transport processes case were significantly lower than that for the decoupled transport processes case. PDI is one of the most important parameters determining the quality of the polymer product, so its realistic prediction is of great practical importance and hence, is highly desirable. The predicted values of DPn did not differ much from each other for decoupled and coupled transport processes cases. Whereas, the predicted values of $P D I$ differed from each other by about $70 \%$ for the two cases, where predicted values for coupled transport processes case were being at lower side.

Figure 6 shows the variation of density at reactor outlet with diffusion coefficient for variable FTPP case. The increase in density with increase in diffusion coefficient was understandable 
from the point of view of corresponding $X_{M}$ as shown in Figure 3. The increase in density along the flow with monomer conversion is important from the fact that increased density would lead to decreased velocity to conserve the mass flow. This would affect both the residence time and residence time distribution inside the reactor as per the reactor geometry. This would not be the case for residence time in case of constant density model.

Figure 7 shows the results for viscosity variation at reactor outlet. This is one of the properties in the polymerization reactions which changes by about $4-6$ orders for full conversion depending on the monomer-polymer system. Besides this, significant changes in viscosity could affect the flow profile as well as mixing and thus heat transfer. Viscosity was found to be increased by 6 orders in our simulations. The viscosity at outlet increased with an increase in diffusion coefficient. This was because of the increased conversion that led to more polymer fraction in the system, which in turn increased the viscosity. As a consequence, the pressure drop across the reactor also increased. Variation of viscosity, pressure drop and density are shown in Figures 8-10 respectively for each reactor geometry. Great variation in density and viscosity clearly proved the importance of modeling their variation and thus proved the necessity of coupling the transport processes with reaction and mixing.

The results for the single case of CFD simulation for all the three reactor geometries with continuously varying diffusion coefficient for various chemical species and constant FTPP are summarized in Table 6.

Here we could observe that the results for $X_{M}$ and $P D I$ were closer to the one predicted by variable FTPP case with discrete variation of diffusion coefficient. On the other hand, DPn was predicted higher and was closer to constant FTPP case. Again the results were found to be independent of the geometry for all the three reactor geometries.

Despite huge variation in viscosity and moderate changes in density, no significant variation of thermal conductivity was observed. The temperature variation across the cross section differed 
only by $0.1 \mathrm{~K}$ even for variable FTPP case simulations. Thus, truly isothermal condition was observed even during high viscosity variation and density changes. So, modeling thermal conductivity as constant would be a good assumption without affecting any results. However, properties like density and viscosity had significant impact on the results of the simulations as aforementioned.

\section{Conclusions}

Three microreactor geometries with gradual change in geometry were taken for study through simulation of FRP under mixed feed condition at reactor inlet. The effects of variation of FTPP case compared to constant FTPP case with discrete variation of diffusion coefficient were studied. This was done to observe the importance of modelling the coupling of transport process with chemical reaction in microreactors. One case of varying diffusion coefficient with constant FTPP was also studied. The significant differences were observed in the predictions of $X_{M}$, $D P n$ and PDI between the variable and constant FTPP cases for the given condition of mixed feed at reactor inlet. The results were found to be closer to physical reality for the variable FTPP case. This clearly established the importance of modeling coupled transport processes with chemical reaction in tubular microreactors. Contrary to expectation, the results obtained were found to be independent of reactor geometries under consideration for either cases. In Appendix-D, the CFIR was shown to exhibit chaotic advection even at such a low inlet Reynolds number of 0.06 thus exhibiting improved mixing. CFIR seemed to be a promising microreactor for polymerization as shown by Parida et al. ${ }^{[25,26]}$ for atom transfer radical polymerization but current study did not get any such results despite having similar (though not same) reaction system. Both the models - the variable FTPP model alongwith discrete variation of diffusion coefficient and Constant FTPP model with continuously varying diffusion coefficient, were definitely an improvement over constant FTPP model with discrete variation of diffusion coefficient. But both could not predict the improved results for CFIR despite having 
improved mixing. Clearly, to evaluate CFIR more thoroughly, a more comprehensive modeling is required. This might include complete variation of FTPP along with continuous variation of diffusion coefficient which would be part of future work.

\section{Supporting Information}

Supporting Information is available from the Wiley Online Library

Appendix-A: Mathematical model for free radical polymerization as used in this work.

Appendix-B: Zhu Transformation and New transformation.

Appendix-C: Expression for the variations in viscosity, density and thermal conductivity for MMA.

Appendix-D: Visualizing mixing inside the three tubular microreactors using mass-less tracer particles

\section{Appendix/Nomenclature/Abbreviations}

$A_{H} \quad$ Area for heat transfer, $m^{2}$

$C_{M} \quad \frac{K_{f m}}{K_{p}}$, dimensionless

$\mathrm{Cp} \quad$ Specific heat capacity of mixture, $\mathrm{cal} / \mathrm{g} /{ }^{\circ} \mathrm{C}$

$C_{T} \quad \frac{K_{t d}}{K_{t c}}$, dimensionless

$D P_{n} \quad$ Number averaged degree of polymerization

I Initiator concentration, $\mathrm{mol} / \mathrm{l}$

$K_{d} \quad$ Dissociation rate coefficient, $\min ^{-1}$

$K_{f m} \quad$ Transfer to monomer rate coefficient, $1 /($ mol.min $)$

$K_{i} \quad$ Kinetic rate constant for initiation, $\mathrm{s}^{-1}$

$K_{p} \quad$ Propagation rate coefficient, 1/(mol.min) 
$K_{p r} \quad K_{p}+K_{f m}=\left(1+C_{M}\right) K_{p}, 1 /($ mol.min $)$

$K_{t} \quad K_{t c}+K_{t d}, 1 /($ mol.min $)$

$K_{t c} \quad$ Termination by combination rate coefficient, $1 /(\mathrm{mol} . \mathrm{min})$

$K_{t d} \quad$ Termination by disproportionation rate coefficient, 1/(mol.min)

$L \quad$ Kinetic chain length, $\frac{K_{p r} M \lambda_{0}}{2 f K_{d} I}$

$\dot{L} \quad L \cdot\left(\frac{1-R_{M M}}{1+R_{P} L}\right)=L \cdot\left(\frac{1-R_{M}}{1+R_{P} L}\right)$

M Monomer concentration, $\mathrm{mol} / \mathrm{l}$

MW Molecular weight, $\mathrm{g} / \mathrm{mol}$

$M W_{n} \quad$ Number averaged chain length of polymer, g/mol

$M W_{w} \quad$ Weight averaged chain length of polymer, $\mathrm{g} / \mathrm{mol}$

PDI Polydispersity index, dimensionless

$P_{n} \quad$ Dead polymer chain length of $n$ no. of monomer units

$R \quad$ Universal gas constant, $1.986 \mathrm{cal} / \mathrm{mol} / \mathrm{K}$

$R_{0} \quad$ Zero order radical obtained from initiator dissociation

$R_{M} \quad \frac{K_{f m}}{K_{p}+K_{f m}}=\frac{K_{f m}}{K_{p r}}=\frac{C_{M}}{1+C_{M}}$

$R_{M M} \quad R_{M}$

$R_{n} \quad$ Live polymer chain length of $n$ no. of monomer units

$R_{T} \quad \frac{K_{t c}}{K_{t c}+K_{t d}}=\frac{K_{t c}}{K_{t}}=\frac{1}{1+C_{T}}$, dimensionless

T Temperature, $\mathrm{K}$

$T_{\text {bath }} \quad$ Temperature of heat sink, $\mathrm{K}$

$U \quad$ Overall heat transfer coefficient, $\mathrm{W} / \mathrm{m}^{2} / \mathrm{K}$

$V_{R} \quad$ Volume of solution at any time t, liter

$V_{R 0} \quad$ Initial volume of solution at $\mathrm{t} 0$, liter 
$X_{M} \quad$ Monomer conversion, dimensionless

f Initiator efficiency, dimensionless

$f_{s} \quad$ Initial Solvent volume fraction, dimensionless

$f_{v} \quad$ Fractional free volume, dimensionless

$t \quad$ Time, $\min$

u Velocity, $\mathrm{m} / \mathrm{s}$

$\Delta H_{P} \quad$ Heat of reaction, cal $/ \mathrm{mol}$

$\beta \quad$ Ratio of solvent volume to non-solvent volume, dimensionless

$\varepsilon \quad$ Volume contraction factor corrected for solvent volume fraction, dimensionless

$\varepsilon_{0} \quad$ Volume contraction factor without solvent volume fraction, dimensionless

$\lambda_{0} \quad$ Zeroth order moment for live polymer chain length distribution, mol/l

$\lambda_{1} \quad$ First order moment for live polymer chain length distribution, mol/l

$\lambda_{2}$ Second order moment for live polymer chain length distribution, mol/1

$\mu_{0} \quad$ Zeroth order moment for dead polymer chain length distribution, mol/l

$\mu_{1} \quad$ First order moment for dead polymer chain length distribution, mol/l

$\mu_{2} \quad$ Second order moment for dead polymer chain length distribution, mol/1

$\rho \quad$ Mixture density, $\mathrm{g} / \mathrm{cm}^{3}$

$\Phi \quad$ Volume fraction, dimensionless

$\eta \quad$ Dynamic viscosity, $\mathrm{cP}$

Subscript

M Monomer

P Polymer

$S \quad$ Solvent

I Initiator 
Acknowledgements: The financial support by ANR grant No. 09-CP2D-DIP² is greatly appreciated.

Keywords: coupled problem, CFD, mixing, free radical polymerization, coiled flow inverter microreactor

[1] J. Y. Kim, R. L. Laurence, Korean J Chem Eng, 1998, 15 (3), 273.

[2] V. Hessel, H. Lowe, F. Schonfeld, Chem Eng Sci, 2005, 60 (8-9), 2479.

[3] M. M. Mandal, C. Serra, Y. Hoarau, K. D. P. Nigam, Microfluid Nanofluid, 2011, 10 (2), 415 .

[4] a) C. Serra, G. Schlatter, N. Sary, F. Schonfeld, G. Hadziioannou, Microfluid Nanofluid, 2007, 3 (4), 451; b) C. Serra, N. Sary, G. Schlatter, G. Hadziioannoua, V. Hessel, Lab Chip, 2005, 5, 966.

[5] P. E. Baillagou, D. S. Soong, Polym Eng Sci, 1985, 25 (4), 212.

[6] D. K. Garg, C. A. Serra, Y. Hoarau, D. Parida, M. Bouquey, R. Muller, Macromol Theor Simul, 2020, https://doi.org/10.1002/mats.202000008.

[7] D. K. Garg, C. A. Serra, Y. Hoarau, D. Parida, M. Bouquey, R. Muller, Microfluid Nanofluid, 2015, 18, 1287.

[8] A. K. Saxena, K. D. P. Nigam, Aiche J, 1984, 30 (3), 363.

[9] V. Kumar, M. Aggarwal, K. D. P. Nigam, Chem Eng Sci, 2006, 61 (17), 5742.

[10] S. A. Berger, L. Talbot, L. S. Yao, Annu Rev Fluid Mech, 1983, 15, 461.

[11] W. R. Dean, J. M. Hurst, Philosophical Magazine Series 7, 1927, 4 (20), 77.

[12] C. M. White, Proc. R. Soc. Lond. A, 1929, 123, 645. 
[13] N. Ramesh, P. K. Davis, J. M. Zielinski, R. P. Danner, J. L. Duda, J Polym Sci Pol Phys, 2011, 49 (23), 1629.

[14] J. S. Vrentas, J. L. Duda, J Polym Sci Pol Phys, 1977, 15 (3), 417.

[15] J. S. Vrentas, J. L. Duda, J Polym Sci Pol Phys, 1977, 15 (3), 403.

[16] D. K. Garg, C. A. Serra, Y. Hoarau, D. Parida, M. Bouquey, R. Muller, Macromolecules, 2014, 47 (14), 4567.

[17] A. Keramopoulos, C. Kiparissides, J Appl Polym Sci, 2003, 88 (1), 161.

[18] J. M. Zielinski, J. L. Duda, Aiche J, 1992, 38 (3), 405.

[19] P. E. Baillagou, D. S. Soong, Chem Eng Sci, 1985, 40 (1), 75.

[20] S. K. Soh, D. C. Sundberg, J Polym Sci Pol Chem, 1982, 20, 1331.

[21] S. K. Soh, D. C. Sundberg, J Polym Sci Pol Chem, 1982, 20, 1345.

[22] D. S. Achilias, C. Kiparissides, J Macromol Sci R M C, 1992, C32 (2), 183.

[23] J. Brandrup, in Polymer Handbook, (Eds: J. Brandrup, E. H. Immergut), Interscience Publishers, New York 1966.

[24] R. H. Shoulberg, J. A. Shetter, J Appl Polym Sci, 1962, 6 (23), S32.

[25] D. Parida, C.A. Serra, D.K. Garg, Y. Hoarau, R. Muller, M. Bouquey, Macromolecular Reaction Engineering, 2014, 8 (8),597.

[26] D. Parida, C.A. Serra, F. Bally, D.K. Garg, Y. Hoarau, F. Bally, R. Muller, M. Bouquey, Macromolecules, 2014, 47 (10), 3282. 


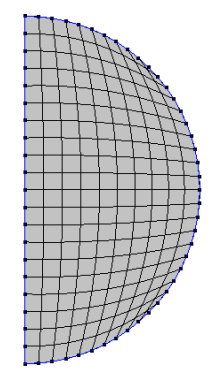

(a)

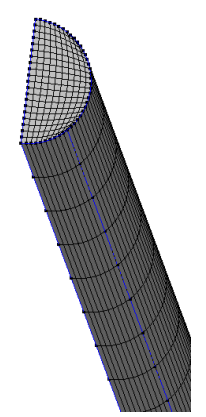

(b)

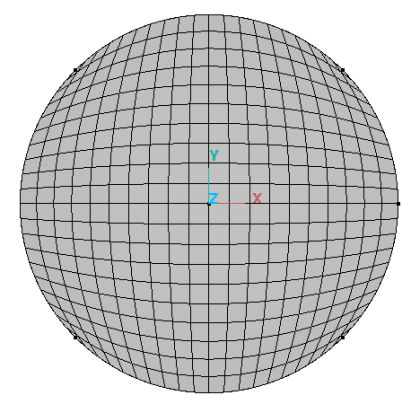

(c)

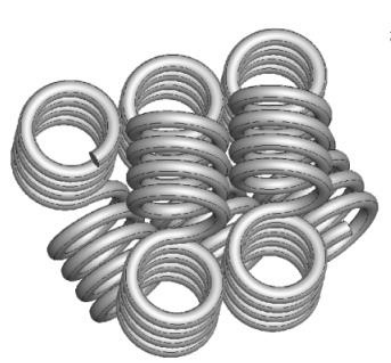

(f)

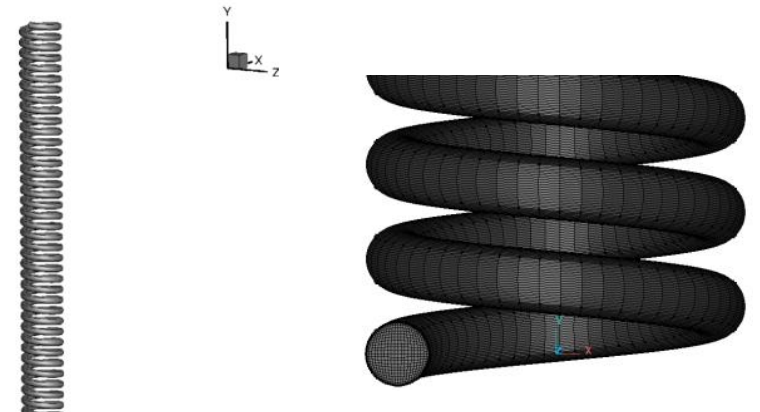

(d)

(e)

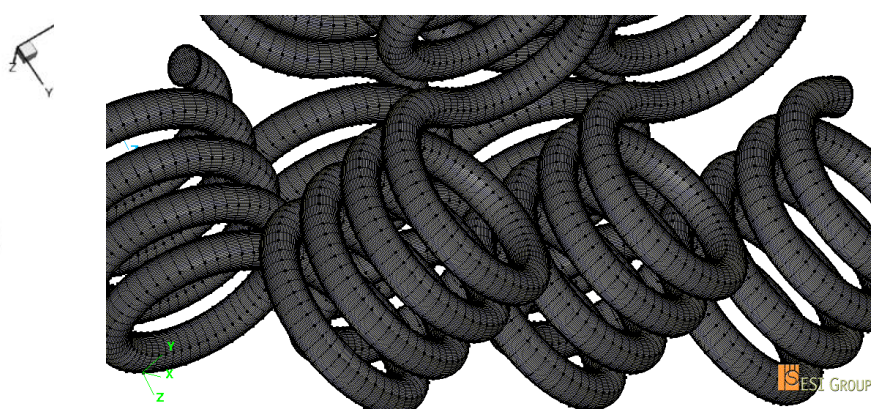

(g)

Figure 1. a) Cross section meshing of STR inlet, b) STR volume grid, c) cross section meshing of CTR and CFIR inlet, d) CTR general view, e) CTR volume grid, f) CFIR general view, g) CFIR volume grid.

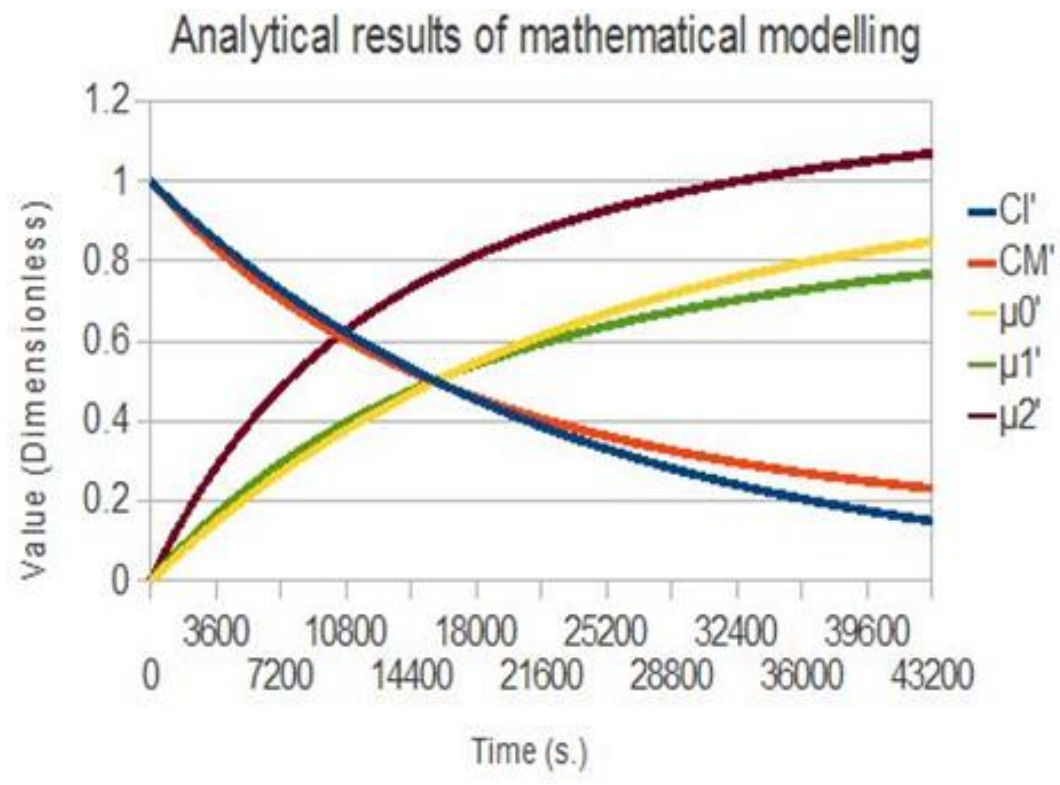

Figure 2. Comparison of results for STR modelled as well mixed isothermal batch reactor with the analytical solution under similar conditions. 


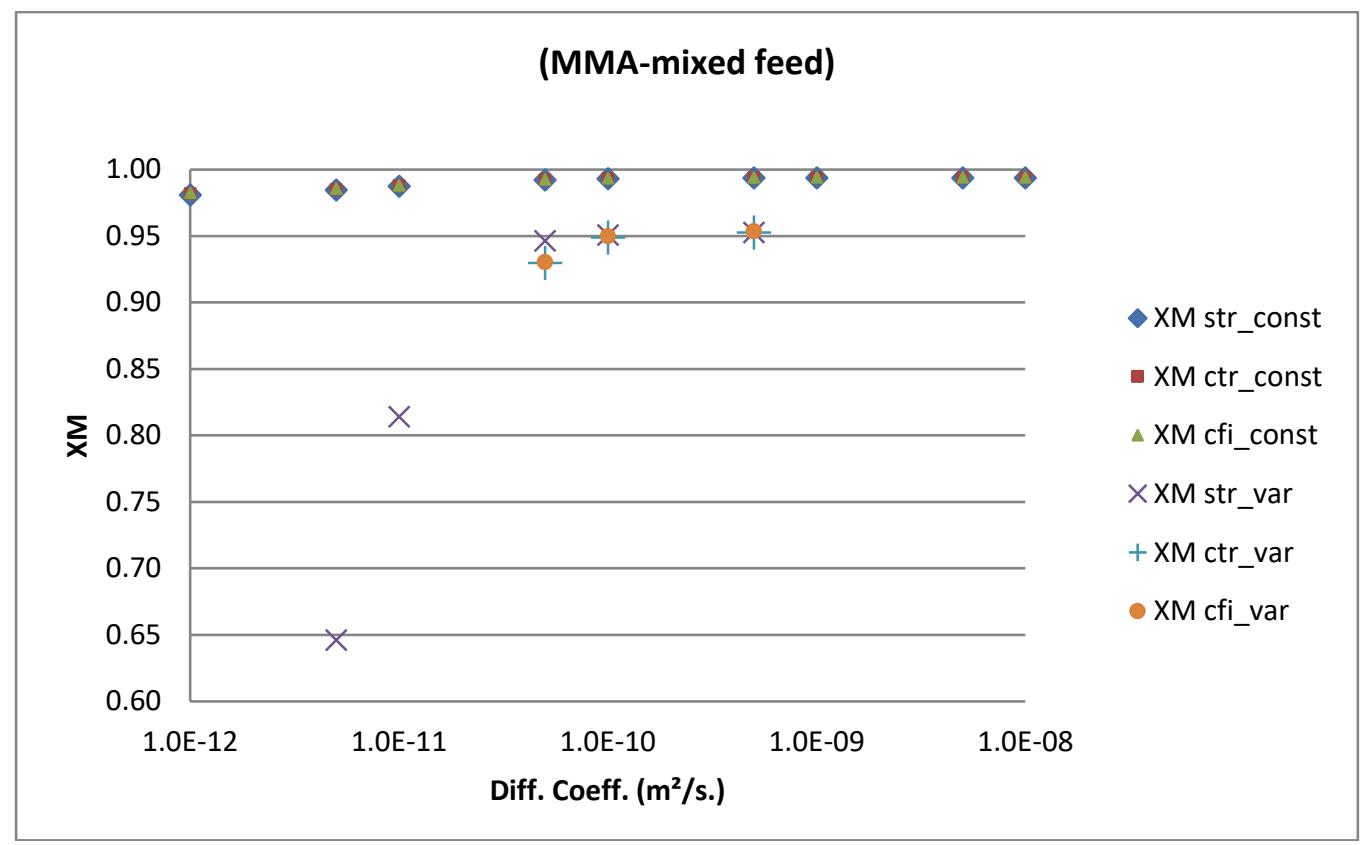

Figure 3. Monomer conversion $\left(X_{M}\right)$ results for STR, CTR and CFIR for constant and variable FTPP.

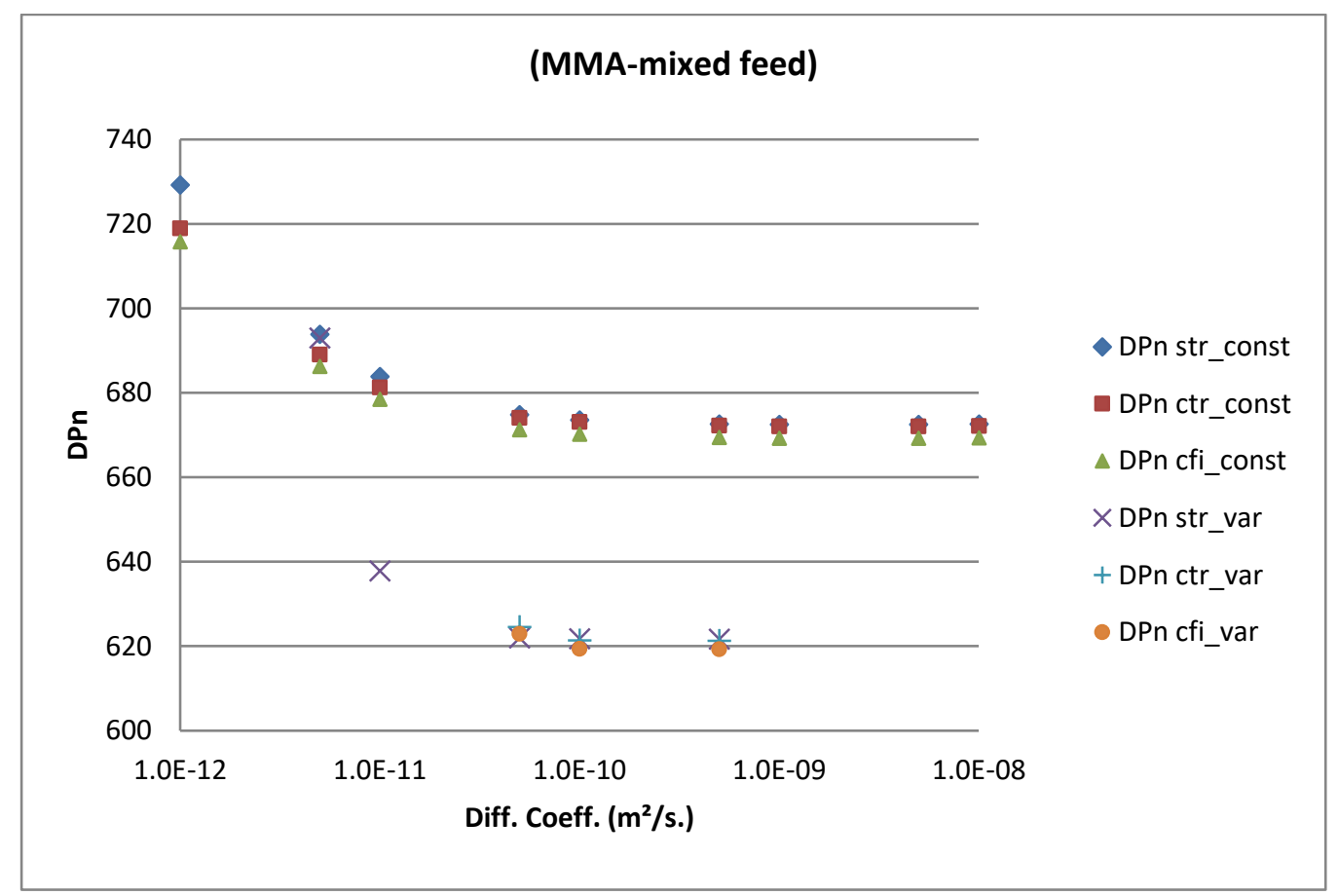

Figure 4. DPn results for STR, CTR and CFIR for constant and variable FTPP cases. 


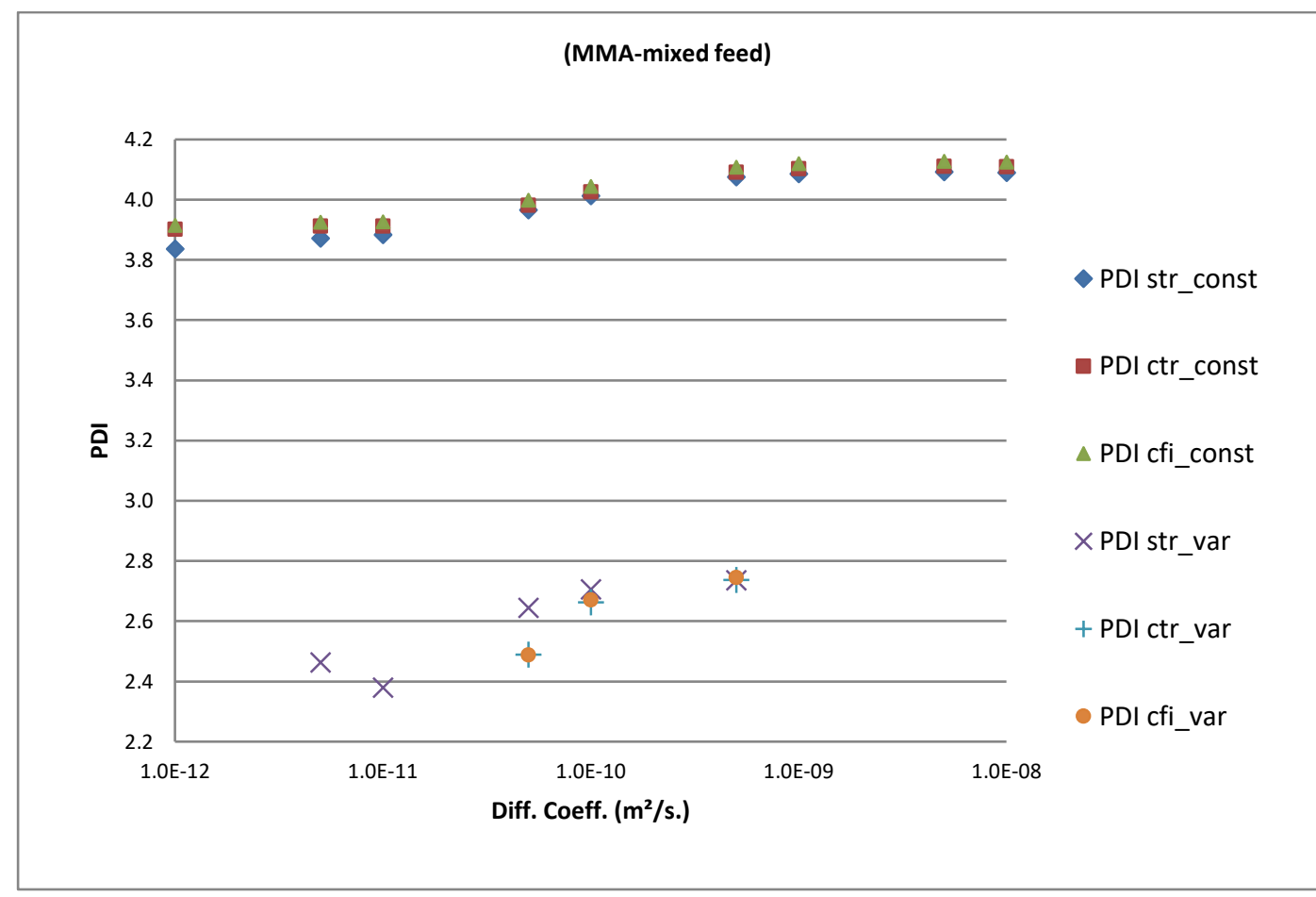

Figure 5. PDI results for STR, CTR and CFIR for constant and variable FTPP

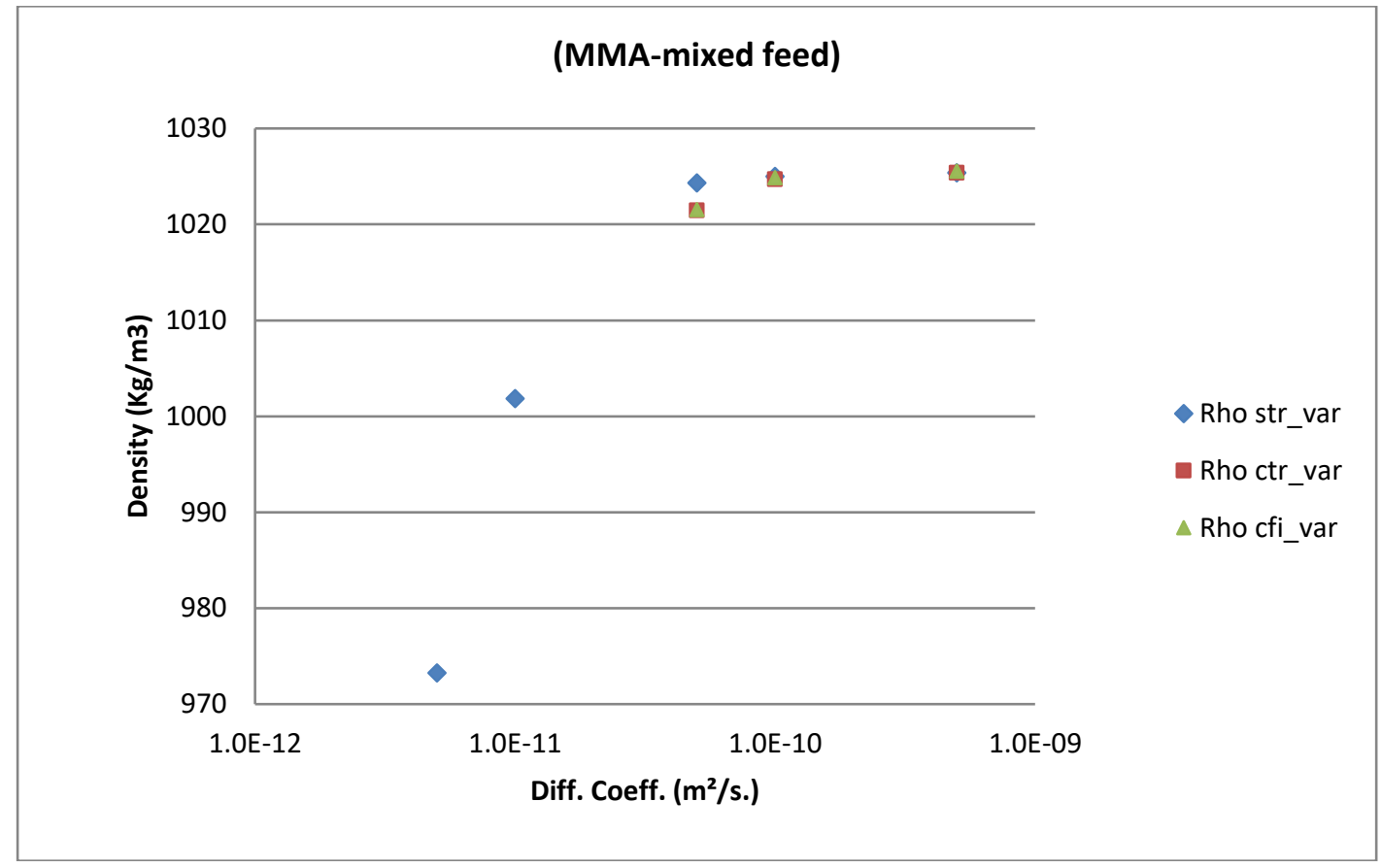

Figure 6. Density results for STR, CTR and CFIR for variable FTPP 


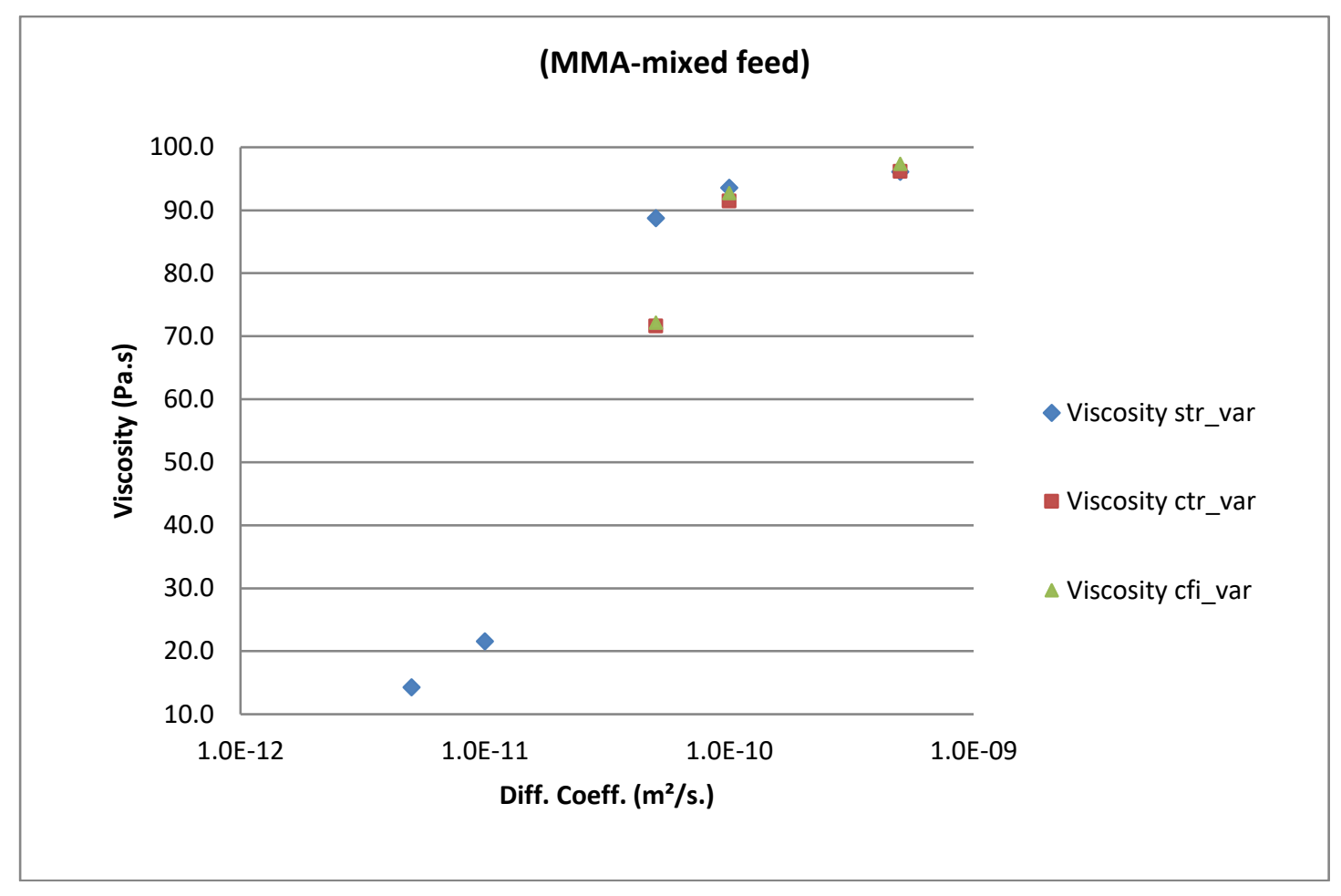

Figure 7. Viscosity results for STR, CTR and CFIR for variable FTPP

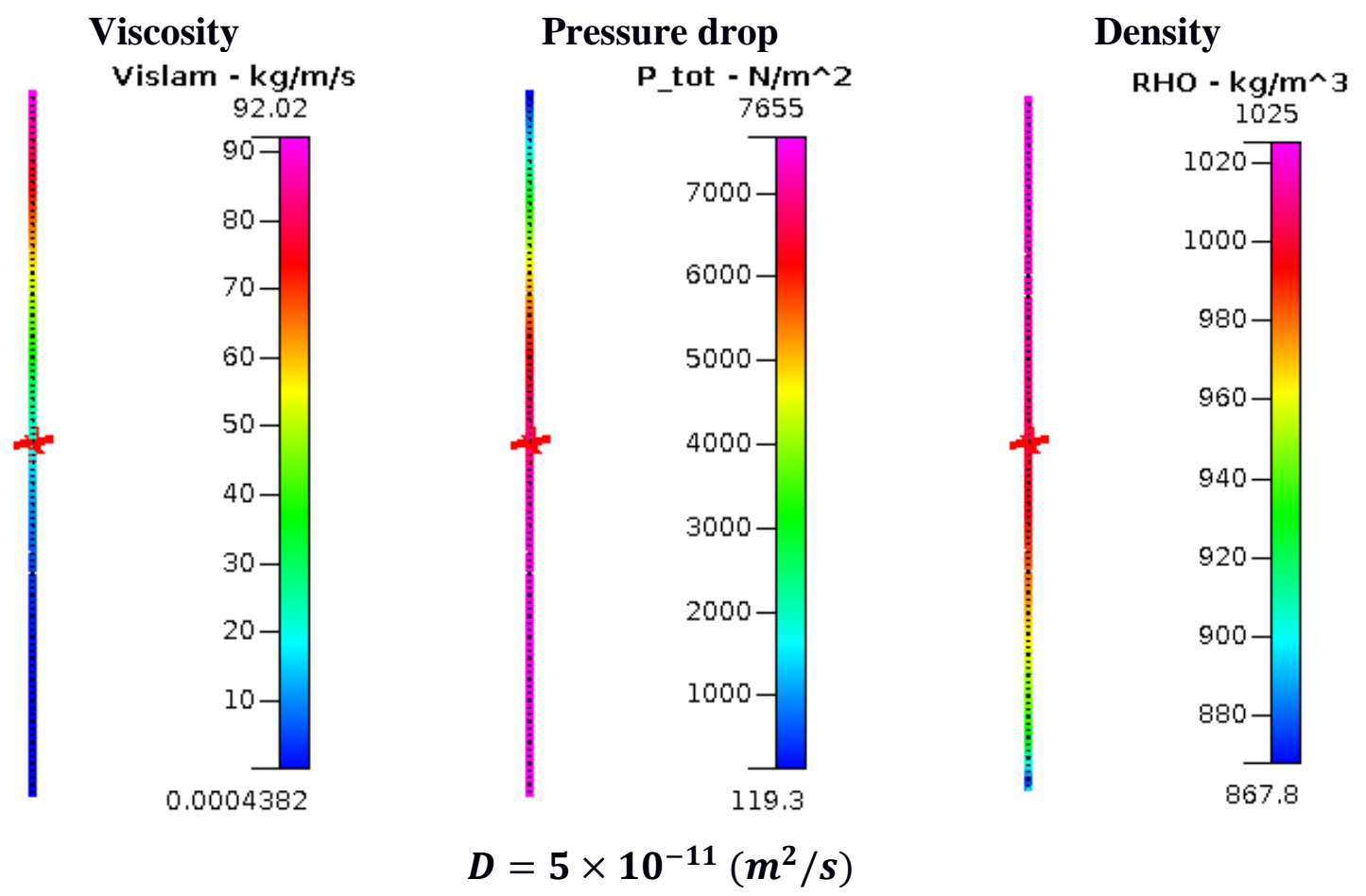

Figure 8. Viscosity, pressure drop and density variation throughout the volume of STR 


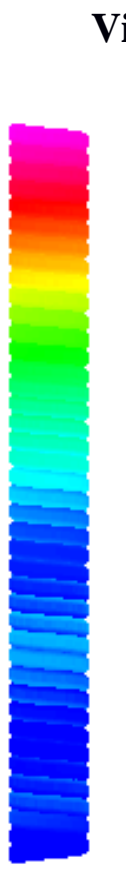

Viscosity

Vislam - $\mathrm{kg} / \mathrm{m} / \mathrm{s}$

76.53

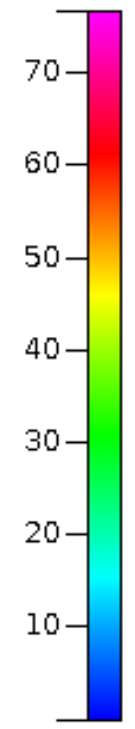

0.000374
Pressure drop

P tot $-\mathrm{N} / \mathrm{m}^{\wedge} 2$

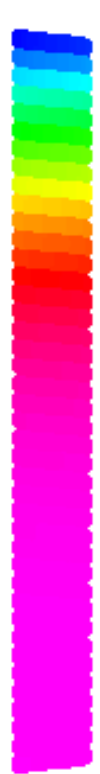

5435

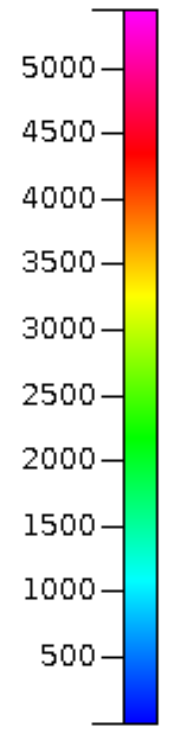

2.641

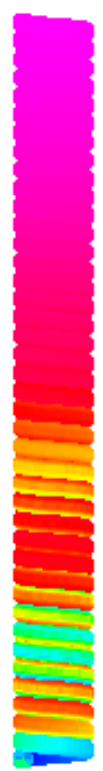

density

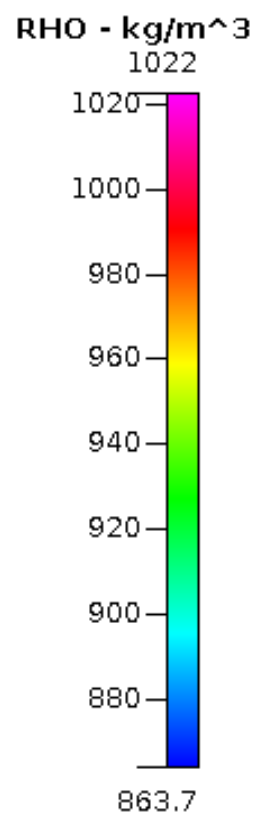

$D=5 \times 10^{-11}\left(m^{2} / s\right)$

P_tot - N/m^

Vislam - $\mathrm{kg} / \mathrm{m}$

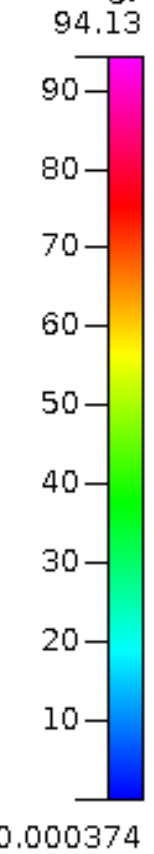

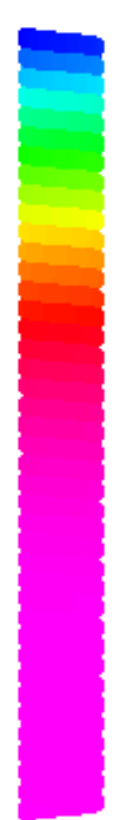

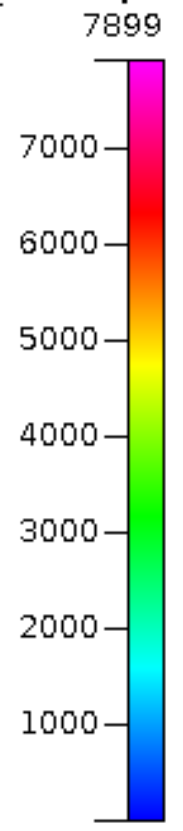

3.288

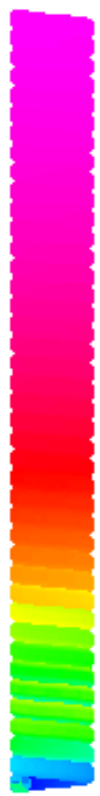

RHO - $\mathbf{k g}_{1025} \mathbf{m}^{\wedge} \mathbf{3}$

$1020-$

$1000-$

$980-$

$960-$

$940-$

$920-$

$900-$

880

863.7

$$
D=1 \times 10^{-10}\left(m^{2} / s\right)
$$

Figure 9. Viscosity, pressure drop and density variation throughout the volume of CTR 

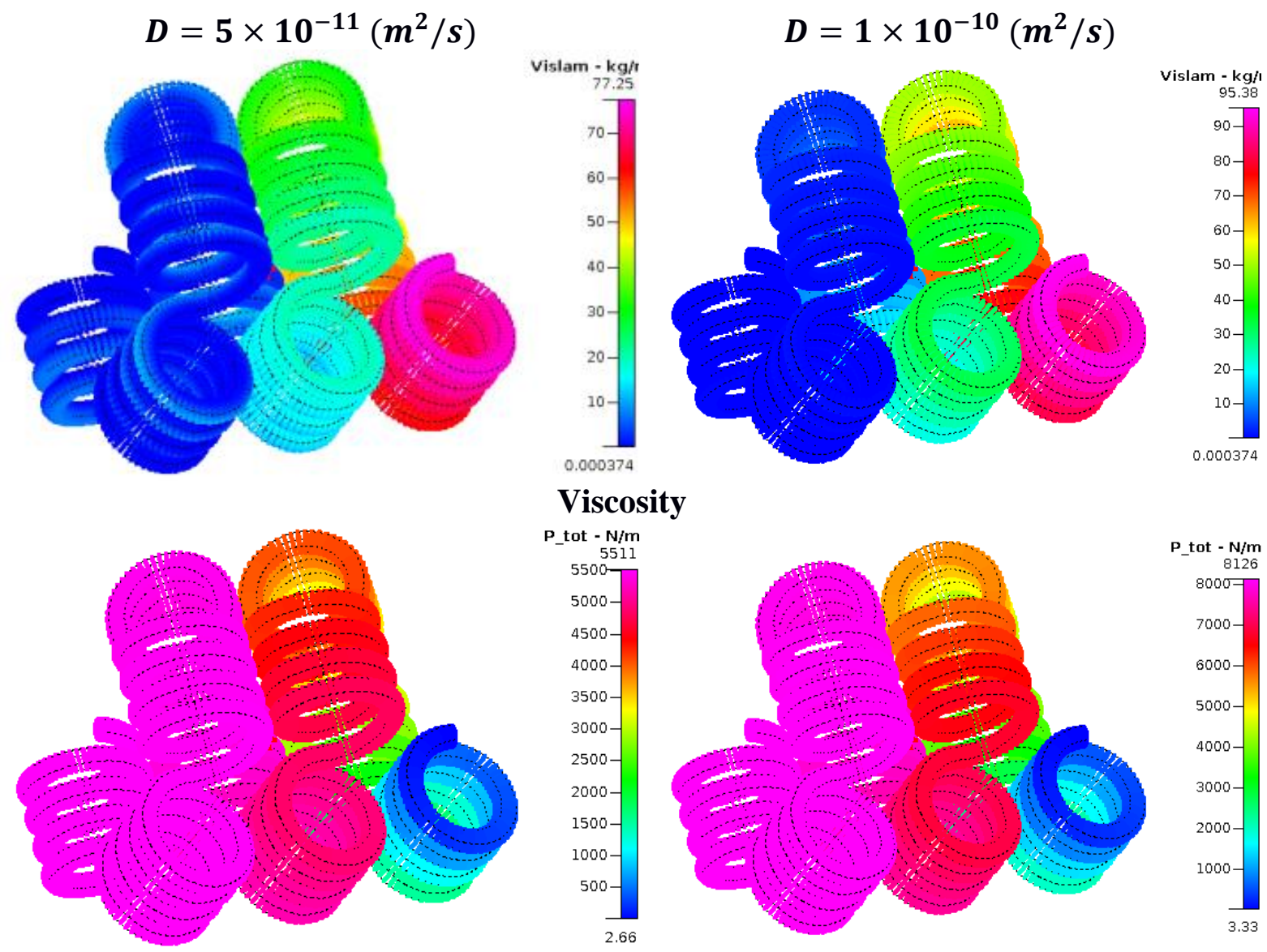

Viscosity
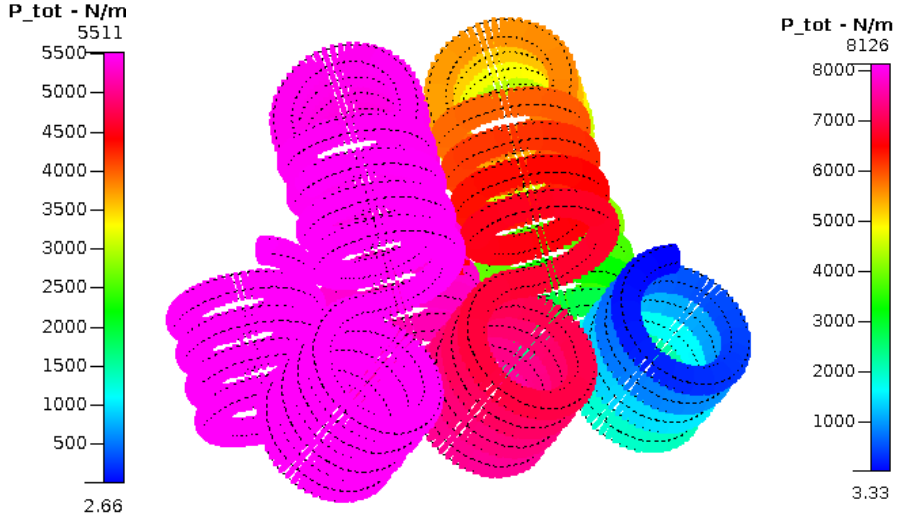

\section{Pressure drop}
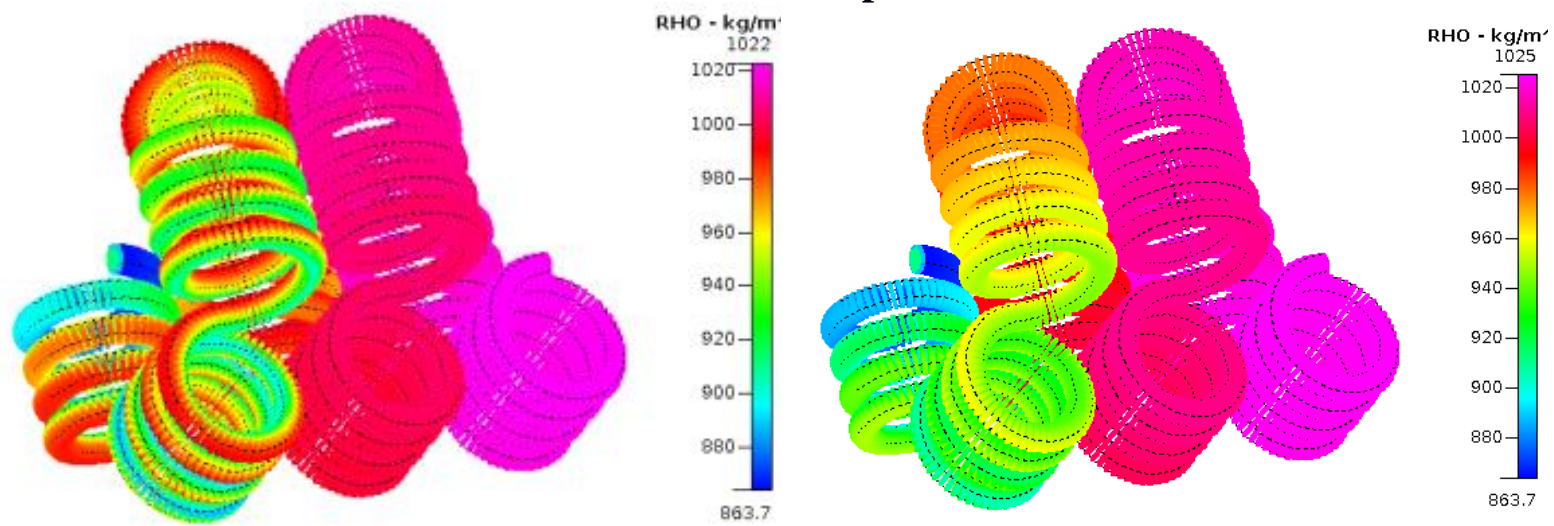

Density

Figure 10. Viscosity, pressure drop and density variation throughout the volume of CFIR 
Table 1. Operating conditions and physical data for constant fluid physical properties simulations $^{5}$

\begin{tabular}{ll}
\hline \multicolumn{1}{c}{ Parameter } & Value \\
\hline Wall temperature $(\mathrm{K})$ - constant & 343.15 \\
\hline Inlet feed temperature $(\mathrm{K})$ & 343.15 \\
\hline Chemical species diffusion coefficient. $\left(\mathrm{m}^{2} / \mathrm{s}\right)$ & from $1 \times 10^{-12}$ to $1 \times 10^{-8}$ \\
\hline Fluid density $\left(\mathrm{kg} / \mathrm{m}^{3}\right)$ & $1 \times 10^{3}$ \\
\hline Fluid viscosity $(\mathrm{Pa} . \mathrm{s})$ & $1 \times 10^{-3}$ \\
\hline Fluid thermal conductivity $(\mathrm{W} / \mathrm{m} / \mathrm{K})$ & 0.6 \\
\hline Fluid specific heat $(\mathrm{J} / \mathrm{kg} / \mathrm{K})$ & 4182 \\
\hline Fluid velocity $(\mathrm{m} / \mathrm{s})$ & $2.9 \times 10^{-5}$ \\
\hline Inlet Reynolds number & 0.06 \\
\hline Residence time $(\mathrm{hrs})$ & 12 \\
\hline
\end{tabular}

Table 2. Reactor data

\begin{tabular}{cccccccc}
\hline $\begin{array}{c}\text { Reactor } \\
\text { Type }\end{array}$ & $\begin{array}{c}\text { Length } \\
(\mathbf{m})\end{array}$ & $\begin{array}{c}\text { Curvature } \\
\text { ratio }^{\text {a) }}\end{array}$ & $\begin{array}{c}\text { No. of } \\
\text { turns }\end{array}$ & $\begin{array}{c}\text { Pitch } \\
(\mathbf{m})\end{array}$ & $\begin{array}{c}\text { Number of } \\
\text { bends }\end{array}$ & $\begin{array}{c}\text { Residence } \\
\text { Time (s) }\end{array}$ & $\begin{array}{c}\text { Excess } \\
\text { length }(\boldsymbol{\%})\end{array}$ \\
\hline STR & 1.252 & - & - & - & 0 & 43200 & 0 \\
\hline CTR & 1.262 & 5 & 40 & 0.003 & 0 & 43546 & +0.8 \\
\hline CFIR & 1.285 & 5 & 40 & 0.003 & 9 & 44340 & +2.6 \\
\hline
\end{tabular}

${ }^{\text {a) }}$ curvature ratio is the ratio between radius of coil and inner radius of tube.

Table 3. Comparison of the data from analytical solution and the simulation of STR as plug flow reactor.

\begin{tabular}{ccc}
\hline & Analytical solution & Plug flow simulation \\
\hline Conversion, $\boldsymbol{X}_{\boldsymbol{M}}$ & 0.770093 & 0.770024 \\
\hline$P D I$ & 1.53879 & 1.53873 \\
\hline$D P n$ & 372.981 & 372.968 \\
\hline
\end{tabular}

Table 4. Mesh independency result for $X_{M}$ for fully mixed inlet to STR.

\begin{tabular}{llllll}
\hline Conversion, $X_{M}$ & Axial, $\mathbf{Z}=\mathbf{1 0}$ & $\mathbf{Z = 5 0}$ & $\mathbf{Z = 1 0 0}$ & $\mathbf{Z = 2 0 0}$ & $\mathbf{Z = 5 0 0}$ \\
\hline $\begin{array}{l}\mathbf{2 0 x 2 0} \\
\text { cross-section) }\end{array}$ & 0.7621 & 0.7681 & 0.7682 & 0.7683 & 0.7683 \\
\hline $\mathbf{3 0 x 3 0}$ & 0.7621 & 0.7681 & 0.7682 & 0.7683 & 0.7683 \\
\hline $\mathbf{5 0 x 5 0}$ & 0.7621 & 0.7681 & 0.7683 & 0.7683 & 0.7683 \\
\hline $\mathbf{1 0 0 x 1 0 0}$ & 0.7621 & 0.7681 & 0.7683 & 0.7683 & 0.7683 \\
\hline
\end{tabular}


Table 5. Result of mesh independency analysis.

Reactor Geometry

Mixed Feed Inlet

\begin{tabular}{ccc}
\hline & Cross section & Flow direction \\
\hline STR & $20 \times 20$ & 100 \\
\hline CTR & $20 \times 20$ & $55^{*}$ \\
\hline CFIR & $20 \times 20$ & $55^{*}$ \\
\hline
\end{tabular}

* For CTR and CFIR, this is the number of points in flow direction in one complete turn. So total number of points $=55 \mathrm{x}$ (number of turns)

Table 6. Results for variable diffusion coefficient with constant thermo-physical properties STR

CTR

CFIR

\begin{tabular}{cccc}
\hline $\boldsymbol{X}_{\boldsymbol{M}}$ & 0.905 & 0.909 & 0.910 \\
\hline $\boldsymbol{P D I}$ & 2.59 & 2.62 & 2.62 \\
\hline $\boldsymbol{D P n}$ & 693 & 685 & 683 \\
\hline
\end{tabular}


Author Photograph(s) $((40 \mathrm{~mm}$ wide $\times 50 \mathrm{~mm}$ high, grayscale $))$

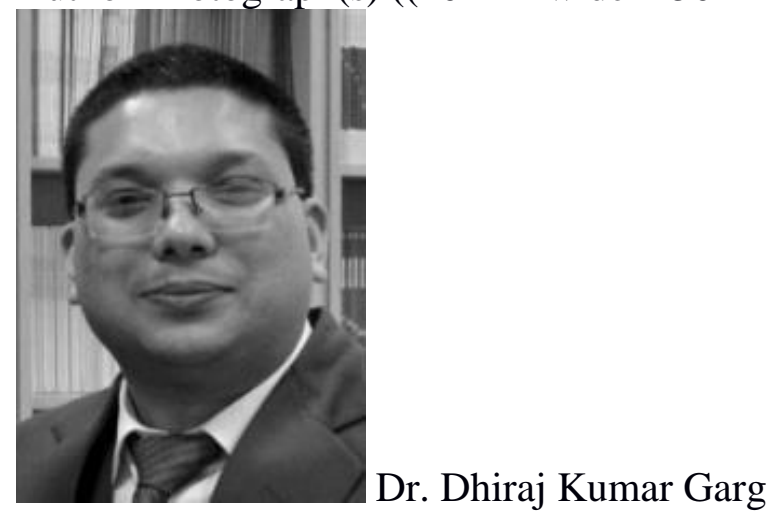

The importance of modeling polymerization with coupled transport processes in microreactor is clearly established in this work. It affects the prediction as well as the trend of various polymer characteristic parameters. Sensitive phenomena like chaotic advection in chaotic microreactor can be observed in a better way while modeling polymerization in it.

Dhiraj K. Garg*, Christophe A. Serra, Yannick Hoarau, Dambarudhar Parida, Michel Bouquey, Rene Muller

Numerical investigations of perfectly mixed condition at the inlet of free radical polymerization tubular microreactors of different geometries

ToC figure

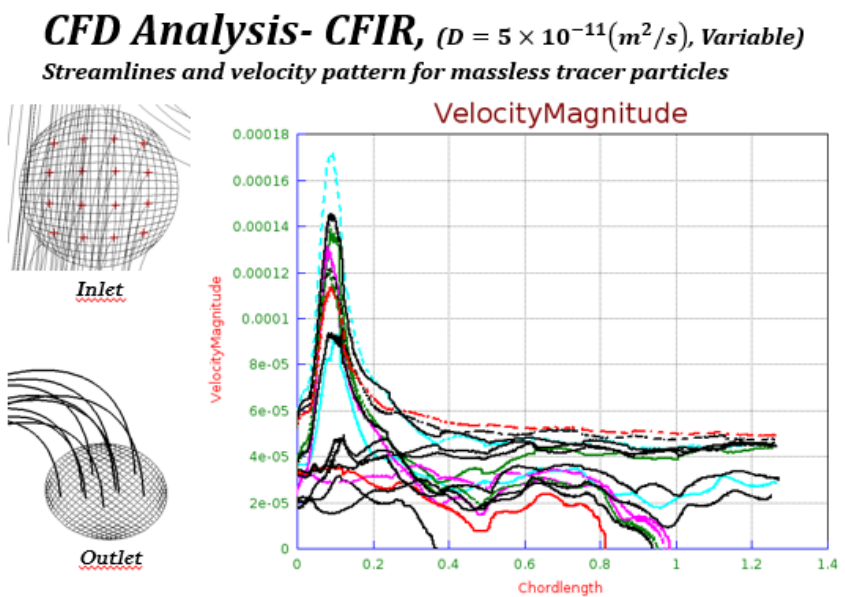




\section{Supporting Information}

\section{APPENDICES}

\section{Numerical investigations of perfectly mixed}

\section{condition at the inlet of free radical polymerization}

\section{tubular microreactors of different geometries}

Dhiraj K. Garg*, Christophe A. Serra, Yannick Hoarau, Dambarudhar Parida, Michel Bouquey, Rene Muller

Appendix-A: Mathematical model for free radical polymerization as used in this work. Initiator decomposition

$\frac{-1}{V_{R}} \frac{d\left(I . V_{R}\right)}{d t}=K_{d} I$

Monomer

$\frac{-1}{V_{R}} \frac{d\left(M \cdot V_{R}\right)}{d t}=\left(K_{p}+K_{f m}\right) M \lambda_{0}=\left(1+C_{M}\right) K_{p} M \lambda_{0}=K_{p r} M \lambda_{0}$

$\frac{d x_{M}}{d t}=\left(K_{p}+K_{f m}\right)\left(1-x_{M}\right) \lambda_{0}=\left(1+C_{M}\right) K_{p}\left(1-x_{M}\right) \lambda_{0}=K_{p r}\left(1-x_{M}\right) \lambda_{0}$

Solvent

$\frac{-1}{V_{R}} \frac{d\left(S \cdot V_{R}\right)}{d t}=K_{f S} S \lambda_{0}=C_{S} K_{p} S \lambda_{0}=R_{S} K_{p r} S \lambda_{0}$

CTA

$\frac{-1}{V_{R}} \frac{d\left(A \cdot V_{R}\right)}{d t}=K_{f a} A \lambda_{0}=C_{A} K_{p} A \lambda_{0}=R_{A} K_{p r} A \lambda_{0}$

Live polymer chains length distribution based on Quasi Steady State Assumption (QSSA)

$\frac{1}{V_{R}} \frac{d\left(\lambda_{0} \cdot V_{R}\right)}{d t}=2 f K_{d} I-\left(K_{t c}+K_{t d}\right) \lambda_{0}^{2}=2 f K_{d} I-K_{t} \lambda_{0}^{2}$ 


$$
\begin{aligned}
& \frac{1}{V_{R}} \frac{d\left(\lambda_{1} \cdot V_{R}\right)}{d t}=2 f K_{d} I+K_{p} M \lambda_{0}+\left(K_{f m} M+K_{f S} S+K_{f a} A\right)\left(\lambda_{0}-\lambda_{1}\right)-\left(K_{t c}+K_{t d}\right) \lambda_{0} \lambda_{1} \\
& 2 f K_{d} I+\left(M+R_{S} S+R_{A} A\right) K_{p r} \lambda_{0}-K_{t} \lambda_{0} \lambda_{1}-\left(R_{M} M+R_{S} S+R_{A} A\right) K_{p r} \lambda_{1} \\
& 2 f K_{d} I+\left(1+R_{S M}+R_{A M}\right) K_{p r} M \lambda_{0}-K_{t} \lambda_{0} \lambda_{1}-\left(R_{M M}+R_{S M}+R_{A M}\right) K_{p r} M \lambda_{1} \\
& \frac{1}{V_{R}} \frac{d\left(\lambda_{2} \cdot V_{R}\right)}{d t}=2 f K_{d} I+K_{p} M\left(2 \lambda_{1}+\lambda_{0}\right)+\left(K_{f m} M+K_{f s} S+K_{f a} A\right)\left(\lambda_{0}-\lambda_{2}\right)-\left(K_{t c}+\right. \\
& \left.K_{t d}\right) \lambda_{0} \lambda_{2} \\
& 2 f K_{d} I+\left(M+R_{S} S+R_{A} A\right) K_{p r} \lambda_{0}+2 K_{p} M \lambda_{1}-K_{t} \lambda_{0} \lambda_{2}-\left(R_{M} M+R_{S} S+R_{A} A\right) K_{p r} \lambda_{2} \\
& 2 f K_{d} I+\left(1+R_{S M}+R_{A M}\right) K_{p r} M \lambda_{0}+2 K_{p} M \lambda_{1}-K_{t} \lambda_{0} \lambda_{2}-\left(R_{M M}+R_{S M}+R_{A M}\right) K_{p r} M \lambda_{2}
\end{aligned}
$$

Dead polymer chain length distribution

$$
\begin{aligned}
& \frac{1}{V_{R}} \frac{d\left(\mu_{0} \cdot V_{R}\right)}{d t}=\left(K_{f m} M+K_{f S} S+K_{f a} A\right) \lambda_{0}+\left(K_{t d}+\frac{K_{t c}}{2}\right) \lambda_{0}^{2} \\
& \left(R_{M} M+R_{S} S+R_{A} A\right) K_{p r} \lambda_{0}+\left(1-\frac{R_{T}}{2}\right) K_{t} \lambda_{0}^{2} \\
& \frac{1}{V_{R}} \frac{d\left(\mu_{1} \cdot V_{R}\right)}{d t}=\left(K_{f m} M+K_{f S} S+K_{f a} A\right) \lambda_{1}+\left(K_{t d}+K_{t c}\right) \lambda_{0} \lambda_{1} \\
& \left(R_{M} M+R_{S} S+R_{A} A\right) K_{p r} \lambda_{1}+K_{t} \lambda_{0} \lambda_{1} \\
& \frac{1}{V_{R}} \frac{d\left(\mu_{2} \cdot V_{R}\right)}{d t}=\left(K_{f m} M+K_{f S} S+K_{f a} A\right) \lambda_{2}+\left(K_{t d}+K_{t c}\right) \lambda_{0} \lambda_{2}+K_{t c} \lambda_{1}^{2} \\
& \left(R_{M} M+R_{S} S+R_{A} A\right) K_{p r} \lambda_{2}+K_{t} \lambda_{0} \lambda_{2}+R_{T} K_{t} \lambda_{1}^{2}
\end{aligned}
$$

Energy balance equation

$\frac{d\left(\rho . C p \cdot V_{R} \cdot T\right)}{d t}=\left(-\Delta H_{P}\right) K_{p} M \lambda_{0} V_{R}-U A_{H}\left(T-T_{b a t h}\right)$

Volume variation with reaction

$\frac{d V_{R}}{d t}=-\varepsilon V_{R 0} \frac{d x_{M}}{d t}$

and

Number average molecular weight $M W_{n}=\frac{\lambda_{1}+\mu_{1}}{\lambda_{0}+\mu_{0}} M W_{M} \approx \frac{\mu_{1}}{\mu_{0}} M W_{M}$

Weight average molecular weight $M W_{w}=\frac{\lambda_{2}+\mu_{2}}{\lambda_{1}+\mu_{1}} M W_{M} \approx \frac{\mu_{2}}{\mu_{1}} M W_{M}$ 
Polydispersity Index PDI $=\frac{M W_{w}}{M W_{n}}=\frac{\left(\lambda_{2}+\mu_{2}\right)\left(\lambda_{0}+\mu_{0}\right)}{\left(\lambda_{1}+\mu_{1}\right)^{2}} \approx \frac{\left(\mu_{2} \cdot \mu_{0}\right)}{\left(\mu_{1}\right)^{2}}$

where

$$
\begin{aligned}
& K_{t}=K_{t c}+K_{t d} \\
& K_{p r}=K_{p}+K_{f m}=\left(1+C_{M}\right) K_{p} \\
& C_{M}=\frac{K_{f m}}{K_{p}} \\
& C_{S}=\frac{K_{f s}}{K_{p}} \\
& C_{A}=\frac{K_{f a}}{K_{p}} \\
& C_{T}=\frac{K_{t d}}{K_{t c}} \\
& R_{T}=\frac{K_{t c}}{K_{t c}+K_{t d}}=\frac{K_{t c}}{K_{t}}=\frac{1}{1+C_{T}} \\
& R_{M}=R_{M M}=\frac{K_{f m}}{K_{p}+K_{f m}}=\frac{K_{f m}}{K_{p r}}=\frac{C_{M}}{1+C_{M}} \\
& R_{S}=\frac{C_{S}}{1+C_{M}}=\frac{K_{f s}}{K_{p r}} \\
& R_{S M}=\frac{C_{S}}{1+C_{M}} \frac{S}{M}=R_{S} \cdot \frac{S}{M} \\
& R_{A}=\frac{C_{A}}{1+C_{M}}=\frac{K_{f a}}{K_{p r}} \\
& R_{A M}=\frac{C_{A}}{1+C_{M}} \frac{A}{M}=R_{A} \cdot \frac{A}{M} \\
& R_{P}=R_{M M}+R_{S M}+R_{A M}=R_{M M}+R_{S A} \\
& R_{S A}=R_{S M}+R_{A M} \\
& \rho=\rho_{M} \Phi_{M}+\rho_{P} \Phi_{P}+\rho_{S} \Phi_{S} \\
& C p=C p_{M} \Phi_{M}+C p_{P} \Phi_{P}+C p_{S} \Phi_{S} \\
& \Phi_{M}=\frac{\left(1-x_{M}\right)}{\left(1-\varepsilon_{0} x_{M}+\beta\right)}=\frac{\left(1-x_{M}\right)}{(1+\beta)\left(1-\varepsilon . x_{M}\right)} \\
& \Phi_{P}=\frac{x_{M}(1-\varepsilon)}{\left(1-\varepsilon_{0} x_{M}+\beta\right)}=\frac{x_{M}(1-\varepsilon(1+\beta))}{(1+\beta)\left(1-\varepsilon \cdot x_{M}\right)}
\end{aligned}
$$




$$
\begin{aligned}
& \Phi_{S}=\frac{\beta}{\left(1-\varepsilon_{0} x_{M}+\beta\right)}=\frac{\beta}{(1+\beta)\left(1-\varepsilon \cdot x_{M}\right)} \\
& V_{R}=V_{R 0}\left(1-\varepsilon \cdot x_{M}\right) \\
& M=M_{0} \frac{\left(1-x_{M}\right)}{\left(1-\varepsilon \cdot x_{M}\right)} \\
& \varepsilon=\frac{\varepsilon_{0}}{1+\beta} \\
& \varepsilon_{0}=\frac{\left(\rho_{P}-\rho_{M}\right)}{\rho_{P}}=1-\frac{\rho_{M}}{\rho_{P}} \\
& \beta=\frac{f_{S}}{\left(1-f_{s}\right)}
\end{aligned}
$$

Meaning of all these symbols are same as commonly used and can be found in notation section. 
Appendix-B: Zhu Transformation and New transformation.

Zhu transformation is as follows

For initiator, $\quad I^{\prime}=\frac{I}{I_{0}}$

For monomer, $\quad M^{\prime}=\frac{M}{M_{0}}$

For solvent, $\quad S^{\prime}=\frac{S}{S_{0}}$

For CTA, $\quad A^{\prime}=\frac{A}{A_{0}}$

$$
\begin{aligned}
& \mu_{0}^{\prime}=\frac{\mu_{0}}{I_{0}} \\
& \mu_{1}^{\prime}=\frac{\mu_{1}}{M_{0}} \\
& \mu_{2}^{\prime}=\frac{\mu_{2}}{\left(M_{0}^{2} / I_{0}\right)}
\end{aligned}
$$

To apply NT, as developed in our previous work, ${ }^{9}$ following assumptions are required:

1. Quasi-steady state assumption (QSSA) for live polymer radicals chain lengths distributions $\lambda_{0}, \lambda_{1}, \wedge \lambda_{2}$ to eqs (A6)-(A8), we obtained:

$\lambda_{0}=\sqrt{\frac{2 f K_{d} I}{\left(K_{t c}+K_{t d}\right)}}=\sqrt{\frac{2 f K_{d} I}{K_{t}}}$

$\lambda_{1}=\lambda_{0}(\dot{L}+1)$

$\lambda_{2}=\lambda_{1}(2 \dot{L}+1)=\lambda_{0}(\dot{L}+1)(2 \dot{L}+1)$

where

$\dot{L}=L \cdot\left(\frac{1-R_{M M}}{1+R_{P} L}\right)=L \cdot\left(\frac{1-R_{M}}{1+R_{P} L}\right)$

$L=\frac{\left(K_{p}+K_{f m}\right) M \lambda_{0}}{2 f K_{d} I}=\frac{K_{p r} M \lambda_{0}}{2 f K_{d} I}$

2. For $L \gg 1$

$\lambda_{1}=\lambda_{0} \dot{L}$

$\lambda_{2}=2 L \lambda_{1}=2 L^{2} \lambda_{0}$

The NT for kinetic rate coefficients is as follows:

For dissociation: $K_{d}^{\prime}=K_{d}$ 
For propagation: $K_{p}^{\prime}=K_{p} \sqrt{I_{0} \cdot M_{0}}$

For termination: $K_{t}^{\prime}=K_{t} M_{0}$

So eqs. (B15) - (B17) constitutes the NTs where all terms marked with (') are dimensionless in terms of concentration. $K_{d}$ does not require any transformation as it is already dimensionless in terms of concentration. All the transfer rate coefficients like transfer to monomer $K_{f m}$, transfer to solvent $K_{f s}$, and transfer to CTA $K_{f a}$ are connected to $K_{p}$ through eq. (A18) to (A21) and (A24) to (A28) in Appendix-A. Similarly, $K_{t}$ is connected to $K_{t c} \wedge K_{t d}$ through eq. (A17), (A22) \& (A23) in Appendix-A. An additional relationship for eq. A29 is required if any of the transfer processes is/are involved which is as follows:

$R_{P}^{\prime}=R_{P} \cdot \frac{M_{0}}{I_{0}}$

Applying NT results into the following relationships between their dimensionless and dimensional forms:

$$
\begin{aligned}
& \lambda_{0}^{\prime}=\sqrt{\frac{2 f K_{d}^{\prime} I^{\prime}}{K_{t}^{\prime}}}=\frac{\sqrt{\frac{2 f K_{d}^{I}}{K_{t}}} * 1}{\sqrt{I_{0} M_{0}}}=\frac{\lambda_{0}}{\sqrt{I_{0} M_{0}}} \\
& L^{\prime}=\frac{K_{p}^{\prime} \lambda_{0}^{\prime} M^{\prime}}{2 f K_{d}^{\prime} I^{\prime}}=\frac{K_{p} \lambda_{0} M}{2 f K_{d} I} *\left(\frac{I_{0}}{M_{0}}\right)=L\left(\frac{I_{0}}{M_{0}}\right) \\
& L^{\prime}=L^{\prime} \cdot\left(\frac{1-R_{M M}^{\prime}}{1+R_{P}^{\prime} L^{\prime}}\right)=L \cdot\left(\frac{1-R_{M}}{1+R_{P} L}\right)=L \\
& D P n=\frac{\mu_{1}}{\mu_{0}}=\frac{\mu_{1}^{\prime}}{\mu_{0}^{\prime}}\left(\frac{M_{0}}{I_{0}}\right) \\
& P D I=\frac{\mu_{0} \mu_{2}}{\mu_{1}^{2}}=\frac{\mu_{0}^{\prime} \mu_{2}^{\prime}}{\mu_{1}^{\prime 2}}
\end{aligned}
$$


Appendix-C: Expression for the variations in viscosity, density and thermal conductivity for MMA.

\section{Viscosity}

$$
\begin{aligned}
& c_{p}=1.2 \Phi_{p} \\
& f_{v}=\left[0.025+10^{-3}\left(T\left({ }^{\circ} \mathrm{C}\right)+106\right)\right] \Phi_{m}+\left[0.025+10^{-3}\left(T\left({ }^{\circ} \mathrm{C}\right)+180\right)\right] \Phi_{s}+[0.025+ \\
& \left.0.48 \times 10^{-3}\left(T\left({ }^{\circ} \mathrm{C}\right)-114\right)\right] \Phi_{p} \\
& \eta_{m}=\exp \left[2.303\left(\frac{0.115}{0.025+10^{-3}\left(T\left({ }^{\circ} \mathrm{C}\right)+106\right)}-1\right)\right] \\
& \eta=\eta_{m}+0.6 c_{p}^{1.4} \exp \left(\frac{0.8}{f_{v}}\right) \operatorname{forc}_{p}<0.13 \mathrm{~g} / \mathrm{cm}^{3} \\
& \eta=\eta_{m}+200 c_{p}^{4.2} \exp \left(\frac{0.8}{f_{v}}\right) \operatorname{forc}_{p}>0.13 \mathrm{~g} / \mathrm{cm}^{3}
\end{aligned}
$$

where $\Phi_{m}, \Phi_{p}, \Phi_{s}$ are volume fraction of monomer, polymer and solvent defined by eqn.(A33)-(A35) respectively, $\eta$ the viscosity is in centipoise, $f_{v}$ the fractional free volume, $c_{p}$ the polymer concentration in $\mathrm{g} / \mathrm{cm}^{3}$ and $\eta_{m}$ a parameter.

\section{Density}

$$
\begin{aligned}
& \rho=\rho_{M} \Phi_{M}+\rho_{P} \Phi_{P}+\rho_{S} \Phi_{S} \\
& \rho_{M}\left(\frac{g}{c m^{3}}\right)=0.968-1.225 \times 10^{-3}(T-293.15) \\
& \rho_{P}\left(\frac{g}{c m^{3}}\right)=1.212-8.45 \times 10^{-4}(T-273.15) \\
& \rho_{S}\left(\frac{g}{c m^{3}}\right)=0.883-9 \times 10^{-4}(T-273.15)
\end{aligned}
$$

where $\rho_{M}, \rho_{P}, \wedge \rho_{S}$ are density of monomer, polymer and solvent respectively. 


\section{Thermal Conductivity}

$k\left(\frac{W}{m \cdot K}\right)=k_{m} w_{m}+k_{s} w_{s}+k_{p} w_{p}$

$k_{m}=\frac{418.6 \times 47.61}{\left(T\left({ }^{\circ} \mathrm{C}\right)+273.15\right)} \times\left[\frac{0.9665-0.0011 T\left({ }^{\circ} \mathrm{C}\right)}{100.12}\right]^{\frac{4}{3}}$

$k_{S}=\frac{418.6 \times 66.70}{\left(T\left({ }^{\circ} \mathrm{C}\right)+273.15\right)}\left[\frac{0.8838-0.00087 T\left({ }^{\circ} \mathrm{C}\right)}{92.14}\right]^{\frac{4}{3}}$

$k_{p}=418.6 \times 4.5 \times 10^{-4}$

where $w_{m}, w_{s}, \wedge w_{p}$ are weight fractions of monomer, solvent and polymer respectively, given by following expressions:

$$
\begin{aligned}
& w_{m}=\frac{1-x_{m}}{1+\beta} \\
& w_{s}=\frac{\beta}{1+\beta} \\
& w_{p}=\frac{x_{m}}{1+\beta}
\end{aligned}
$$

where $\beta$ is given by eqn. (A40). 
Appendix-D: Visualizing mixing inside the three tubular microreactors using mass-less tracer particles

For visualizing the impact of variation in density and viscosity on flow profile inside the reactor, a mass-less tracer particles technique as available in CFD-VIEW was used. These particles were numerically injected at various positions uniformly spaced at the inlet cross-section. The results are presented for STR, CTR and CFIR in Figures 11 to 17.

In Figure 11, on $\mathrm{x}$-axis, chordlength represented the distance along the flow from the reactor inlet. y-axis represented velocity magnitude of the individual tracer particle at different $x$, i.e. different distances along the flow inside reactor. Different velocities for different particles at $x=0$, were due to parabolic velocity profile (fully developed flow) imposed at inlet. It could easily be seen that for constant FTPP case, the particles followed straight streamlines as expected. For variable FTPP case, the streamlines bended due to the variation in density and viscosity as shown in Figure 12. This bending of streamlines occurred because of following reason. As soon as the monomer-initiator solution entered the STR, reaction started. But the residence time was higher near the wall due to no-slip boundary condition. This led to higher conversion near the wall. Thus viscosity and density increased more near the wall. This constricted the flow through the cross section. This caused deceleration for the flow near the wall and acceleration for the central flow initially. Meanwhile at the central region of the flow, the reaction mixture experienced a lower residence time and hence lower conversion. Consequently, the viscosity was low initially but as it experienced more residence time, conversion increased and thus viscosity and density also increased. This led to its deceleration. But still there was no radial mixing due to convective process as no particle crosses each other's path.

This was not the case for CTR and CFIR. In Figure 13 for CTR, the velocity profiles for various particles along the flow were quite random for both constant and variable FTPP cases because of the secondary flow induced due to curvature. After certain length along the flow, the velocity 
profile for all the particles fluctuated within a smaller range for constant FTPP (decoupled transport processes) case. This range of fluctuation along with fluctuation got further narrowed down for variable FTPP (coupled transport processes) case. The streamlines of the tracer particles at the outlet could be seen in Figure 14.

Different velocity profiles were obtained for different values of the diffusion coefficient. This could be explained as follows. Different values of conversions at outlet were achieved for different values of diffusion coefficients and thus different values of viscosities and densities were spatially observed along the flow. Due to no-slip boundary condition at the wall, the residence time was higher near the wall leading to higher conversion. This increased viscosity near the wall. Along the flow, this envelop of high viscosity expanded towards the center of the tube from the wall. Thus the area available for the low-viscosity flow was reduced along the flow. This could be observed in Figure 14 (b) and Figure 14 (c). The same observation was made by Baillagou et al. ${ }^{[5]}$ too. No particle in this simulation reached zero velocity, hence it could be deduced that no particle reached the wall for the constant FTPP case where the velocity should be equal to zero because of no-slip condition. Since these particles were modeled as massless tracer particles, if they reached the wall and attained zero velocity, they could not move further as the simulation was steady state. The interpretation of this situation in physical terms is as follows. These tracer particles would represent volume packets in real flow. So, if these tracer particles would reach the wall, it would mean that the volume packets with different residence times would reach the wall. Once they reached the wall, they would need to replace stagnant volume packets from there near the wall and in this process would attain zero velocity themselves. Thus, this process would bring volume packets with higher residence time to mainstream. Hence, this would improve radial mixing and would narrow down the residence time distribution. For the case of constant FTPP case, Figure 14 (a) shows no tracer particle reaching zero velocity. But for variable FTPP case, a few tracer particles did reach zero velocity in the beginning when viscosity was low as shown in Figure 14 (b). At later stage, both Figure 
14 (b) and (c) show not much radial mixing. Thus, variable FTPP modeling predicted more radial mixing in CTR compared to constant FTPP case which was closer to reality. However, both types of modeling predicted a relatively poor radial mixing overall in CTR.

For CFIR as shown in Figure 15, the results were quite different compared to CTR. CFIR was designed to be a chaotic device. ${ }^{[12]}$ The chaos being introduced by the $90^{\circ}$ bend at regular intervals as shown in Figure 1. The lead to chaotic advection ${ }^{[27-29]}$ as explained later. So, despite having the same injection location at the inlet as in CTR, the velocity profiles for different massless tracer particles along the flow was totally different. Each bend changed the velocity profile despite such a low inlet Reynolds number which decreased further along the path in variable FTPP case (due to increase in viscosity). This was quite remarkable from the fact that due to this phenomenon, even at such a low Reynolds number and thus low Dean number, more tracer particles compared to CTR reached the wall starting from first to second bend itself. This indicated better radial mixing capability of CFIR. Because of this, only some of the injected particles reached the reactor outlet as could be seen in Figure 16. This indicated better radial mixing capability of CFIR compared to CTR and STR under similar operating conditions. The number of particles reaching outlet decreased for the lower value of diffusion coefficient and increased for the higher value of diffusion coefficient in both coupled and decoupled transport processes case.

In chaotic advection, two particles, even if they were very close to each other at the beginning, when start moving along the flow followed totally different paths ultimately. i.e. they could not be traced back to their original starting positions. This has been shown in Figure $\mathbf{1 7}$ for both constant and variable FTPP cases. Generally chaotic advection is obtained for unsteady state flow but in our case it was observed even in steady state case. This is consistent with the published results that a non-viscous steady-state 3D flow could be chaotic ${ }^{[27]}$ under certain conditions. The whole region of flow might not have chaotic advection depending upon operating conditions. For non-chaotic region of flow, the two massless tracer particles placed 
very near to each other would follow the similar paths and hence would have the same velocity profile. Chaotic advection, thus, could be observed only at certain points of entry to inlet. In Figure 17, the first column shows the injection location of the tracer particles at inlet whereas the second column showed the velocity profiles of particles along the path. Figure 17(a) shows that the two tracer particles reaching the wall at two different distances for the constant FTPP case. In Figure 17(b), one of the particles reached the wall while other reached the outlet showing the chaotic advection. Same result was observed for variable FTPP case in Figure 17(c). As could be seen that the velocity profiles for both the tracer particles remained nearly same for some length along the flow initially. But after first bend and second bend, the profiles changes completely indicating chaos.

[27] H. Aref, J Fluid Mech, 1984, 143 (Jun), 1.

[28] H. Aref, Philos T Roy Soc A, 1990, 333 (1631), 273.

[29] H. Aref, Phys Fluids, 2002, 14 (4), 1315. 

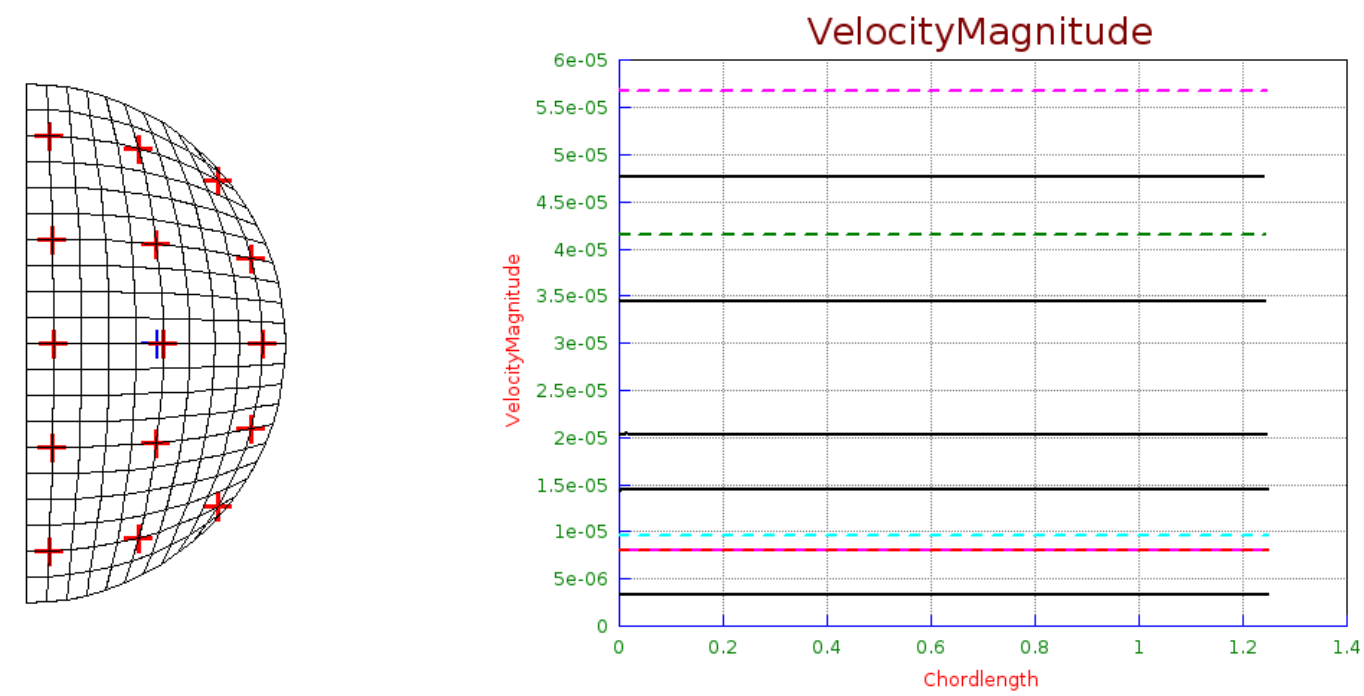

Particles location $5 \times 10^{-11} \mathrm{~m}^{2} / \mathrm{s}$, constant

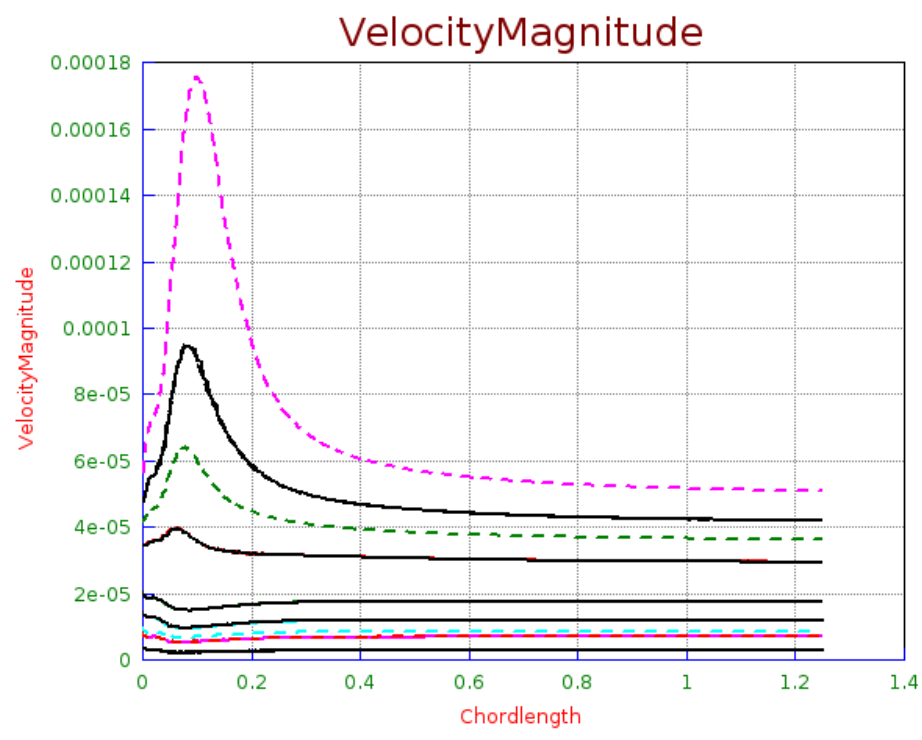

$5 \times 10^{-11} \mathrm{~m}^{2} / \mathrm{s}$, variable

Figure 11. Velocity profile of massless tracer particles in STR for constant or variable FTPP and at a given value of diffusion coefficient

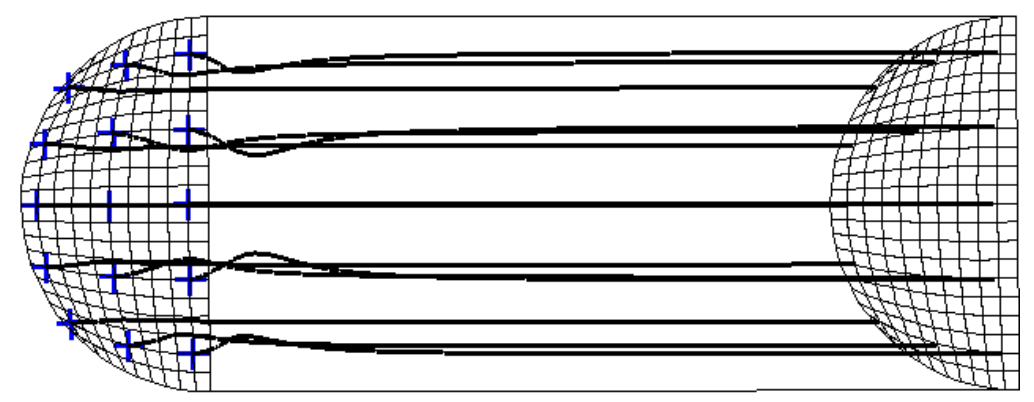

Figure 12. Streamlines of tracers in STR variable FTPP (left is inlet with blue dots for tracers origin and right is outlet of STR) 


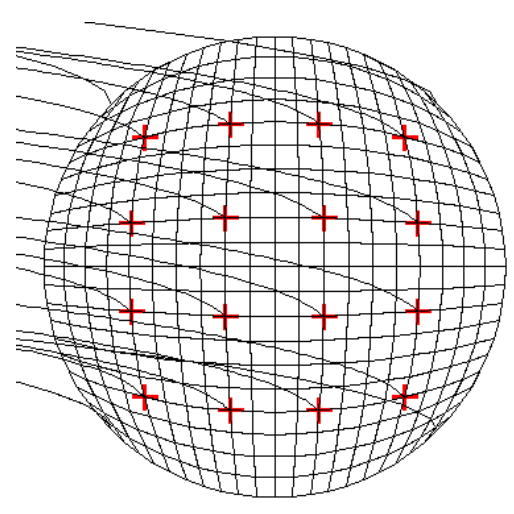

Particles location

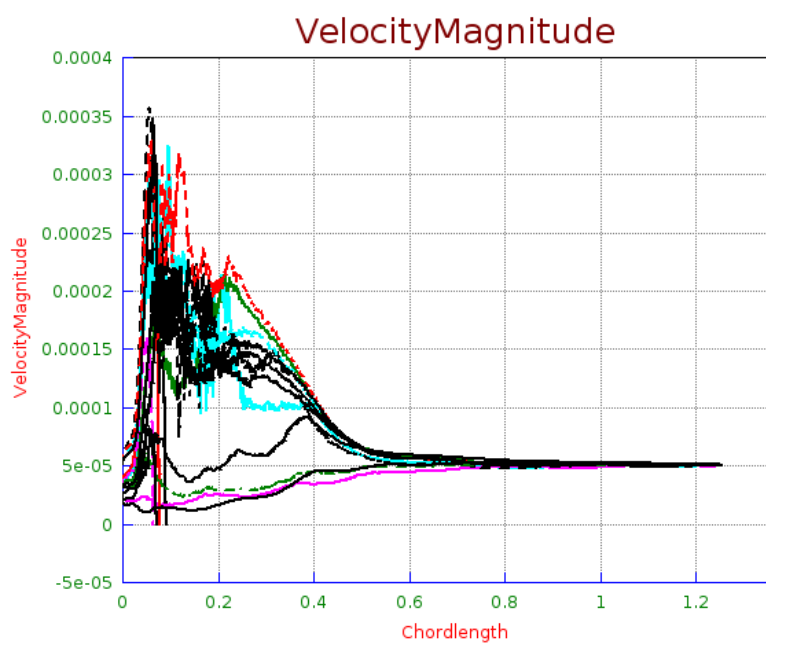

$5 \times 10^{-11} \mathrm{~m}^{2} / \mathrm{s}$, variable

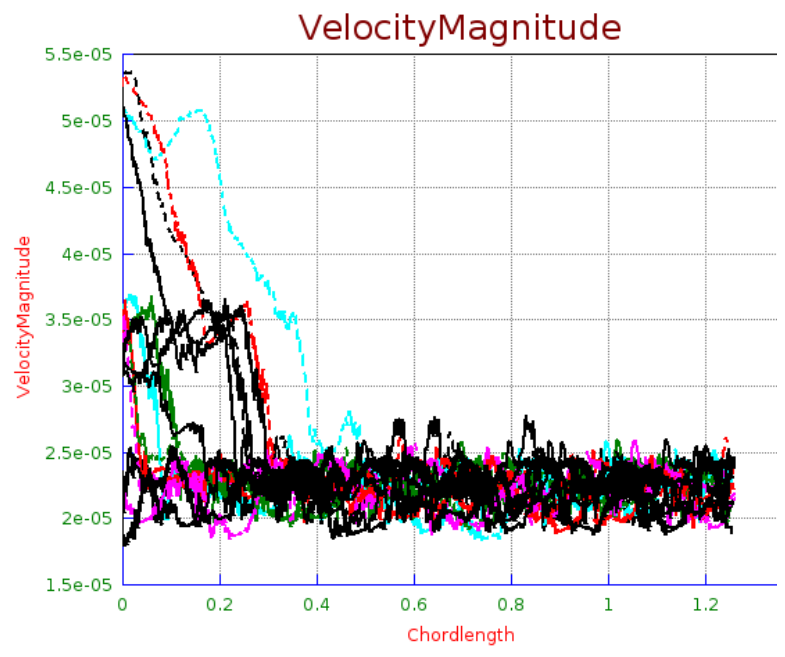

$5 \times 10^{-11} \mathrm{~m}^{2} / \mathrm{s}$, constant

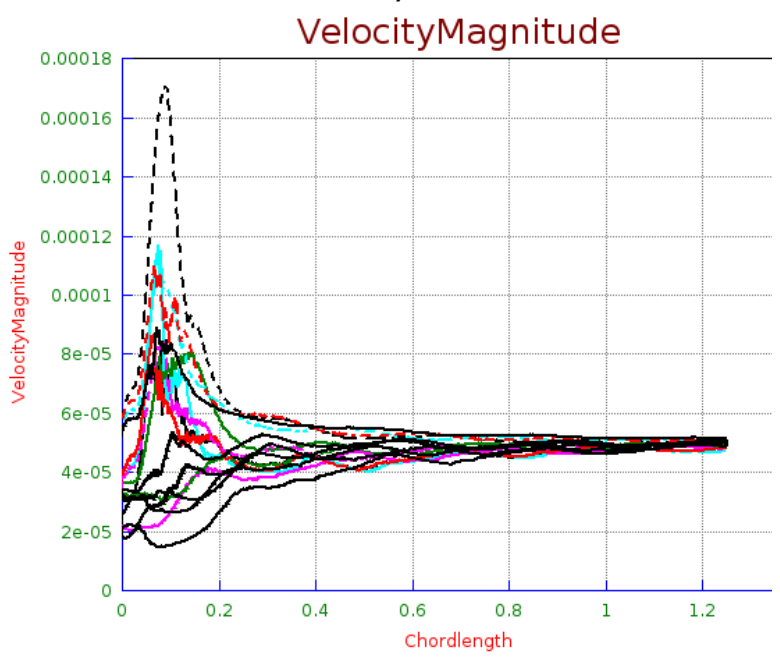

$1 \times 10^{-10} \mathrm{~m}^{2} / \mathrm{s}$, variable

Figure 13. Velocity profile of massless tracer particles in CTR for constant or variable FTPP and at two different values of diffusion coefficient

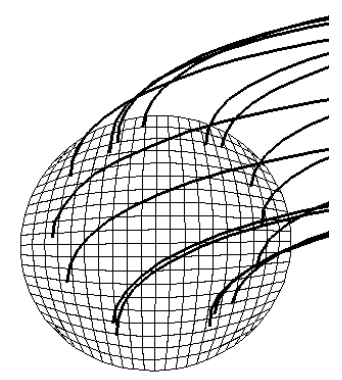

(a)

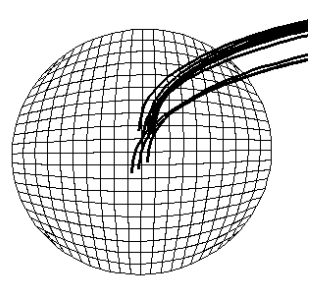

(b)

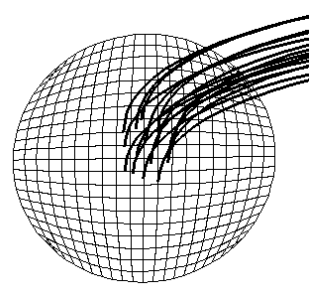

(c)

Figure 14. Streamlines of tracers at CTR outlet for a) constant, b) variable fluid property cases at $D=5 \times 10^{-11}\left(\mathrm{~m}^{2} / \mathrm{s}\right)$, and c) $D=1 \times 10^{-10}\left(\mathrm{~m}^{2} / \mathrm{s}\right)$. 


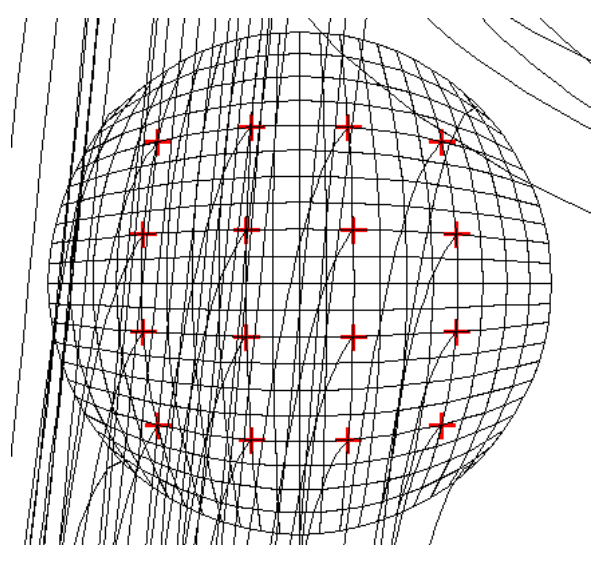

Particles location

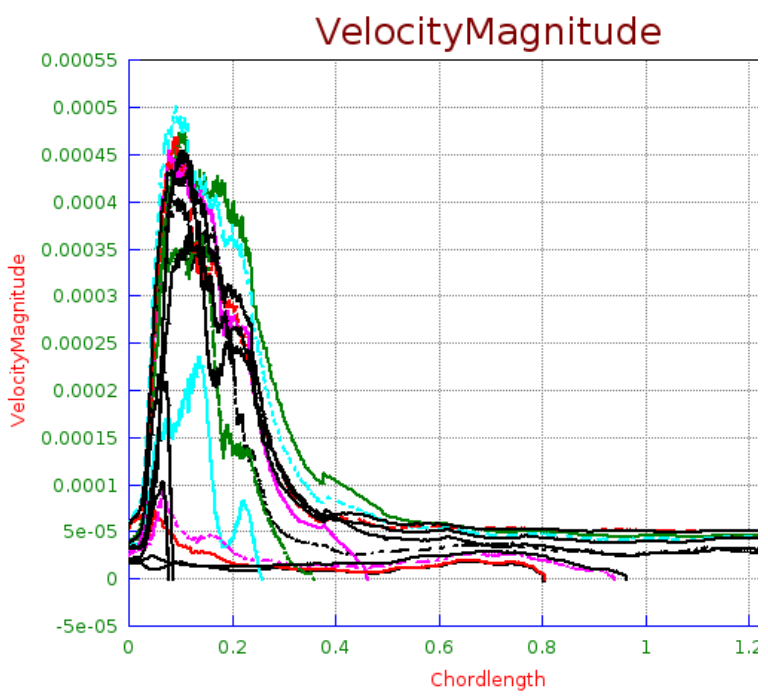

$5 \times 10^{-11} \mathrm{~m}^{2} / \mathrm{s}$, variable

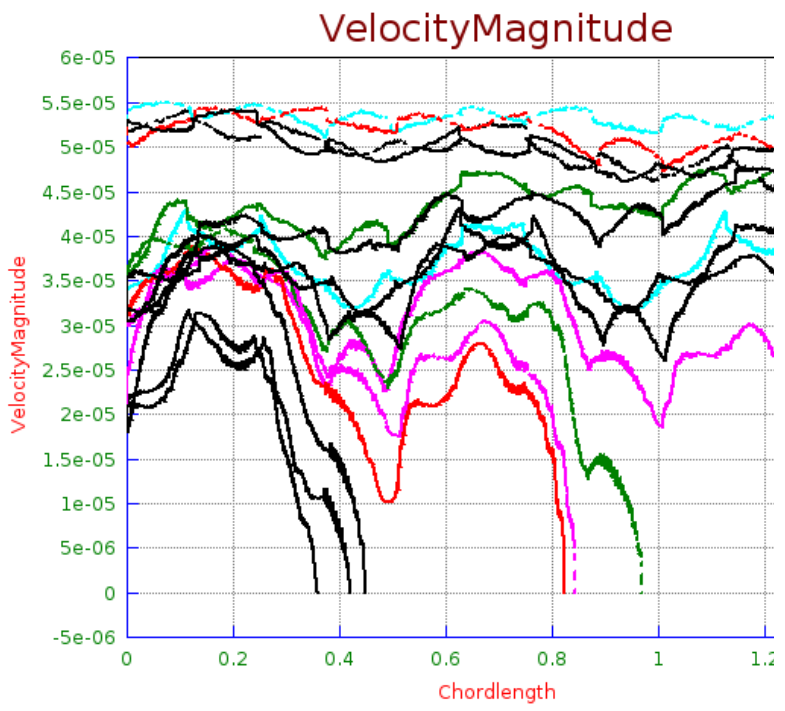

$5 \times 10^{-11} \mathrm{~m}^{2} / \mathrm{s}$, constant

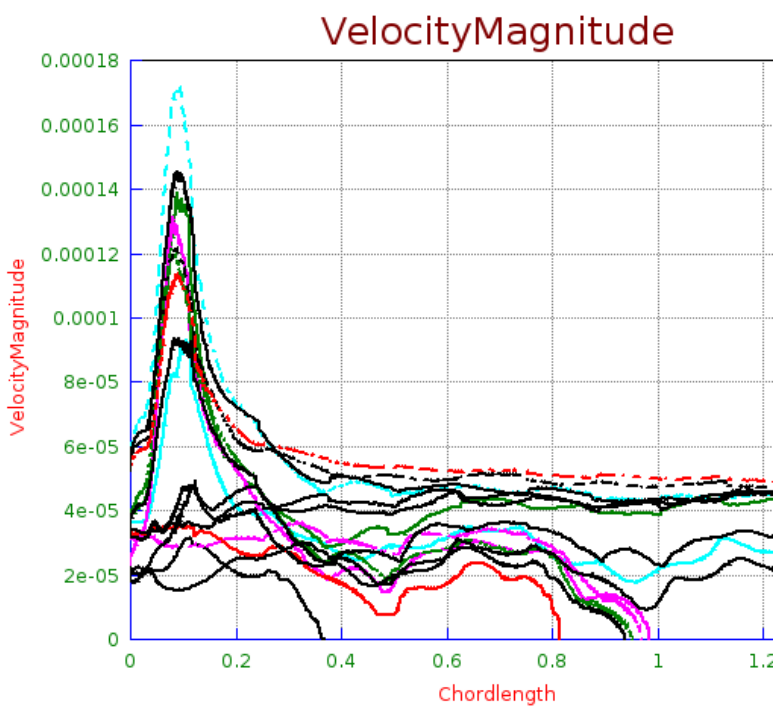

$1 \times 10^{-10} \mathrm{~m}^{2} / \mathrm{s}$, variable

Figure 15. Velocity profile of massless tracer particles in CFIR for constant or variable FTPP and at two different values of diffusion coefficient

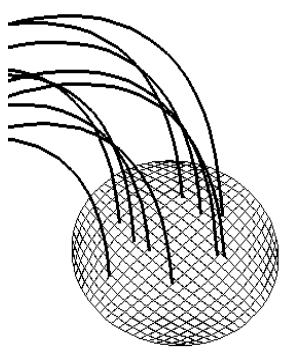

(a)

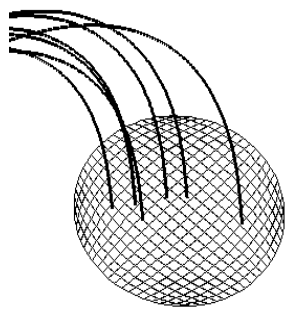

(b)

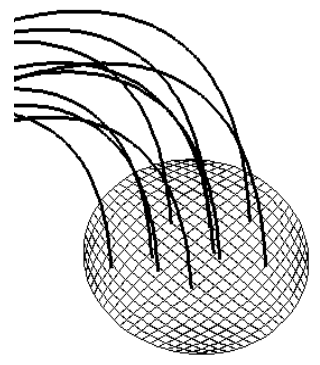

(c)

Figure 16. Streamlines of tracers at CFIR outlet for a) constant, b) variable fluid property cases at $D=5 \times 10^{-11}\left(\frac{m^{2}}{s}\right)$, and c) $D=1 \times 10^{-10}\left(\frac{m^{2}}{s}\right)$ 


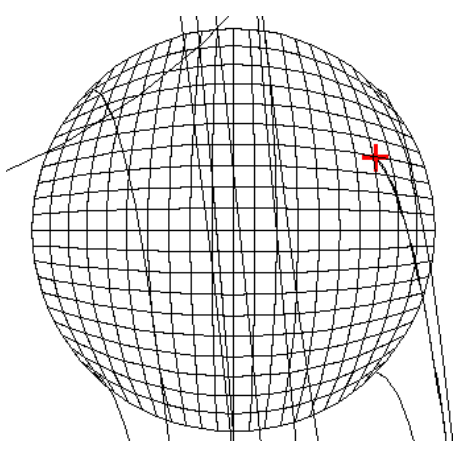

Particles location

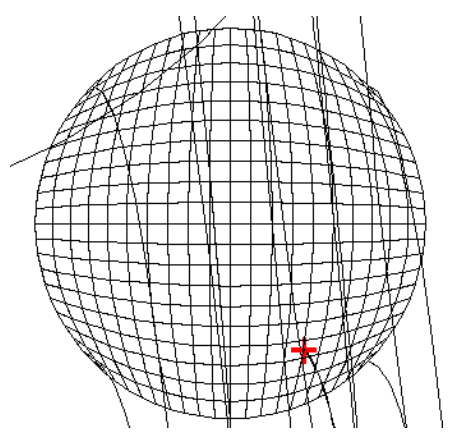

Particles location

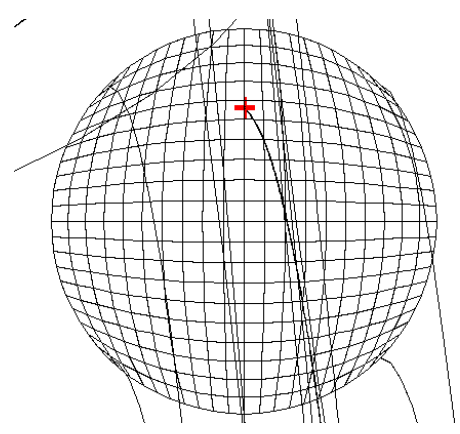

Particles location

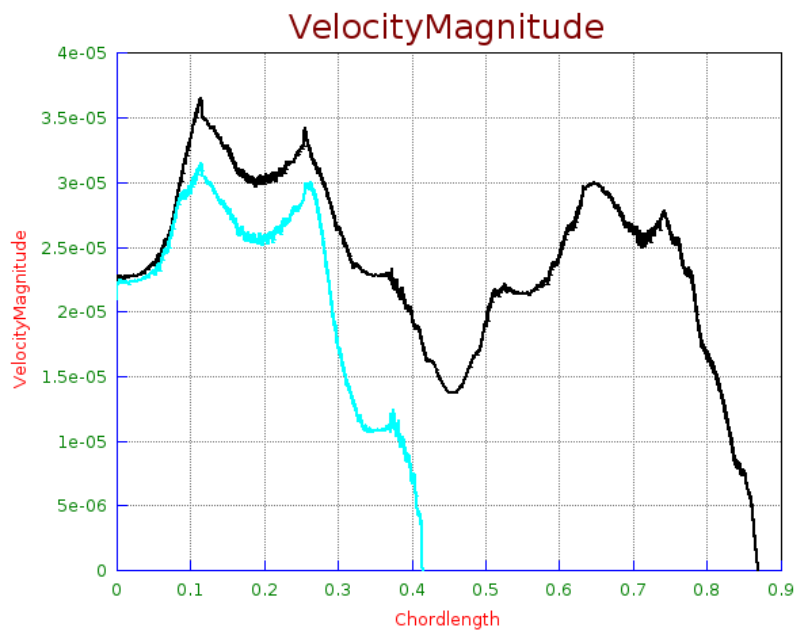

(a) $5 \times 10^{-11} \mathrm{~m}^{2} / \mathrm{s}$, constant

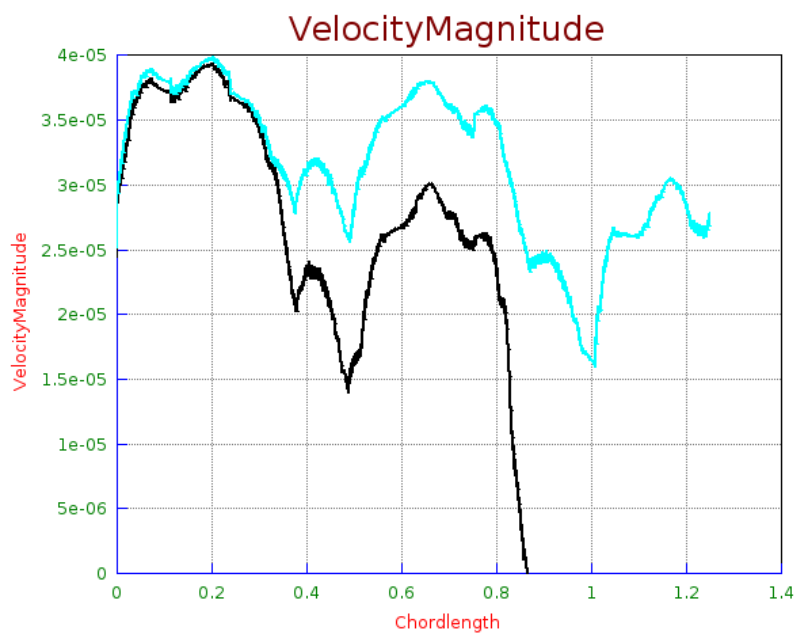

(b) $5 \times 10^{-11} \mathrm{~m}^{2} / \mathrm{s}$, constant

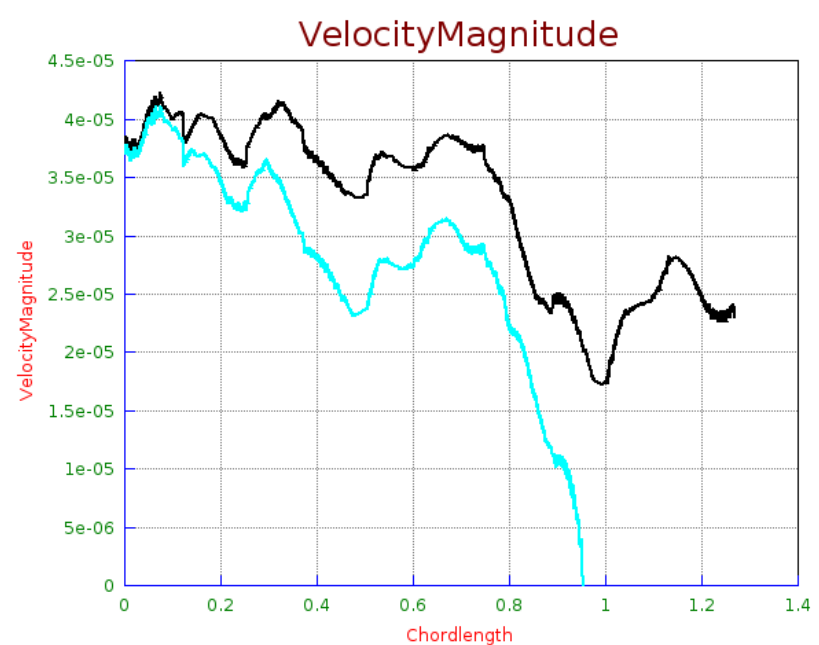

(c) $5 \times 10^{-11} \mathrm{~m}^{2} / \mathrm{s}$, variable

Figure 17. Results showing chaotic trend for two nearly spaced particles at CFIR inlet 